\title{
LISTA DE ESPECIES Y DISTRIBUCIÓN DE LOS ISÓPODOS (CRUSTACEA: PERACARIDA) DE CUBA
}

\section{Species list and distribution of the isopods (Crustacea: Peracarida) from Cuba}

\author{
Manuel Ortiz ${ }^{1}$ y Rogelio Lalana ${ }^{2}$
}

\begin{abstract}
${ }^{1}$ Laboratorio de Crustáceos, Facultad de Estudios Superiores Iztacala, UNAM, México. ortiztouzet@yahoo.com. ${ }^{2}$ Centro de Investigaciones Marinas. Universidad de La Habana, Cuba. rlalana@cim.uh.cu.
\end{abstract}

\section{RESUMEN}

Se ofrece una lista de los isópodos peracáridos de Cuba: 57 géneros y 118 especies marinas y estuarinas, mientras que de los terrestres se presentan 47 géneros y 76 especies registradas. Se actualiza la lista de las especies marinas y estuarinas, al tiempo que se ofrecen anotaciones sobre la ecorregión, localidad específica, sustrato y fecha de colecta de cada una. Esta ha sido preparada con los datos compilados por los autores durante más de 45 años de colectas, separación y clasificación de estos crustáceos peracáridos. La lista de las especies terrestres se basa, principalmente, en los resultados obtenidos durante el desarrollo de las expediciones rumano-cubanas, de los años 70, publicados en revistas de limitado acceso, junto a los resultados posteriores de varios especialistas cubanos. Las ecorregiones más importantes por el número de especies citadas son la noroccidental (Norte Habana-Matanzas) y la Suroccidental (Archipiélago de los Canarreos), mientras que en las ecorregiones Suroriental (Costa Sur de Oriente), Surcentral (Archipiélago Jardines de la Reina) y Nororiental (Costa Norte de Oriente), las citas son nulas. Los isópodos marinos cubanos más comunes son: Cirolana parva, Paracerceis caudata, Limnoria platycauda, Dynamenella perforata y Rocinela signata. El género más común en que se agrupan las especies terrestres es Pseudarmadillo, con 16 especies. Finalmente, se anexa la bibliografía cubana sobre el grupo no citada en el texto.

Palabras clave: isópodos cubanos, lista taxonómica, distribución.

\section{ABSTRACT}

A list of the Cuban peracarid isopods is given: 57 genera and 118 marine and estuarine species, while of the terrestrial ones 47 genera and 76 registered species are presented. The list of marine and estuarine species is updated, while annotations are given on the ecoregion, specific location, substrate and date of collection of each one. This has been prepared with the data compiled by the authors during more than 45 years of collection, separation and classification of these peracarid crustaceans. The one of the terrestrial species is based, mainly, on the results obtained during the development of the Romanian-Cuban expeditions, of the 70s, published in limited access journals, together with the later results of several Cuban specialists. The most important ecoregions for the number of species mentioned are the north-western (North Havana-Matanzas) and the south-western (Canarreos archipelago), while in the ecoregions South-east (South coast of the east), South-central (Archipelago Jardines de la Reina) and Northeast (North Coast of the east), the appointments are null. The most common Cuban marine isopods are: Cirolana parva, Paracerceis caudata, Limnoria platycauda, Dynamenella perforata and Rocinela signata. The most common genus of terrestrial species is Pseudarmadillo with 16 species. Finally, the Cuban bibliography on the group not cited in the text is annexed. 


\section{INTRODUCCIÓN}

Los isópodos constituyen un grupo de crustáceos peracáridos sumamente abundantes, caracterizados generalmente por presentar el cuerpo comprimido dorso-ventralmente. Se distribuyen desde los grandes abismos marinos hasta las aguas someras, y en la tierra firme desde la línea de la costa hasta las altas montañas. También pueden ser localizados como integrantes de la fauna hipogea. Existen especies espongícolas, otras sin embargo, viven entre los vegetales marinos, el arrecife de coral, los fondos arenosos y sobre las raíces del mangle rojo. Algunos son parásitos de peces, de crustáceos decápodos o de otros invertebrados. También ciertos grupos taladran la madera introducida en el mar, provocando, a veces, grandes estragos en los muelles y los cascos de las embarcaciones. Las especies marinas, en la región, han mostrado siempre una gran predilección por los fragmentos de coral muerto o "coral rubble" (Ortiz y Cházaro Olvera, 2017).

Su tamaño es pequeño, generalmente menores de $1 \mathrm{~cm}$. Su taxonomía es complicada, a tal punto que, para las identificaciones correctas, casi siempre se hace necesaria la disección de sus piezas bucales, pereópodos, pleópodos y urópodos.

\section{OBJETIVO}

-Compilar y actualizar la información existente sobre la taxonomía, distribución de especies y la bibliografía de los isópodos de Cuba.

\section{MATERIALES Y MÉTODOS}

El ordenamiento taxonómico se ha estructurado alfabéticamente, utilizando el sistema de clasificación de Ahyong et al. (2011). Todas las especies citadas han sido validadas en WoRMS (2016) y en Boyko et al. (2018).

Las nueve ecorregiones referidas en el presente trabajo han sido las establecidas por Areces (2012) y son las siguientes: 1- Suroriental (Costa Sur de Oriente); 2- Sur Central (Archipiélago Jardines de la Reina); 3- Sur Central (Costa al sur del Macizo de Guamuhaya); 4- Suroccidental (Archipiélago de los Canarreos), 5- Suroccidental (Península de Guanahacabibes); 6- Noroccidental (Archipiélago de Los Colorados); 7- Noroccidental (Norte Habana-Matanzas); 8- Norcentral (Archipiélago Sabana-Camagüey); 9- Nororiental (Costa Norte de Oriente), ver Fig. 1.

Las especies terrestres y troglobias han sido relacionadas dentro de las 15 provincias y el municipio especial Isla de la Juventud, de acuerdo con el accidente geográfico correspondiente. Cuando en la distribución de las especies aparece la palabra Cuba, indica que no se han logrado obtener las citas de sus localidades en el país.

Se anexa la bibliografía que hace referencia a especies cubanas del grupo no citadas en el texto (Anexo 1). 


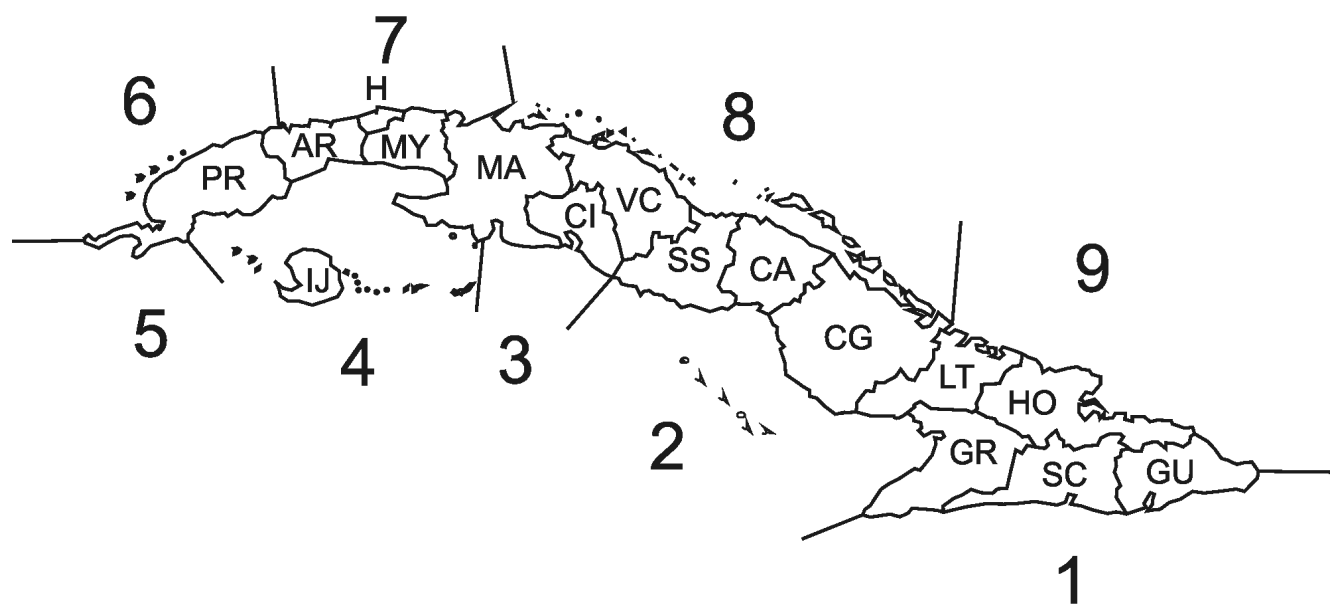

Figura 1. Mapa de Cuba con las nueve ecorregiones (números 1-9) y su distribución en provincias. PR, Pinar del Río; AR, Artemisa; H, La Habana; MY, Mayabeque; MA, Matanzas; CI, Cienfuegos; VC, Villa Clara; SS, Sancti- Spíritus; CA, Ciego de Ávila; CG, Camaguey; LT, Las Tunas; HO, Holguín; GR, Gramma; SC, Santiago de Cuba; GU, Guantánamo; IJ, Isla de la Juventud.

\section{RESULTADOS}

\section{Recuento histórico}

La primera referencia sobre especies cubanas del grupo que nos ocupa es el trabajo de Saussure (1857), no consultado. Después, otros especialistas hicieron alguna referencia a estos crustáceos cubanos. Una de las primeras en hacerlo fue Richardson (1901), citando para Cuba las especies: Aega antillensis Shioedte y Meinert, 1879; Aega dentata Shioedte y Meinert, 1879-80; Rocinella cubensis Richardson, 1898; Rocinella dummerilli (Lucas, 1849); Anilocra laticauda Milne Edwards, 1840 (actualmente nomen nudum); Cymothoa excisa; Cubaris murinus Brandt, 1833 y Ligia baudiniana Milne Edwards, 1840.

La misma autora, en su obra Monograph of the isopods of North America, agrega siete especies más de isópodos para Cuba: Aega tenuipes Schioedte y Meinert, 1879-80; Cirolana cubensis Hay, 1903; Livoneca redmanii Leach, 1818; Ligia exotica Roux, 1828; Pseudoarmadillo carinulatus Saussure, 1857; Pseudoarmadillo gillianus Richardson, 1902 y Porcellio laevis Latreille, 1804 (Richardson, 1905).

Años más tarde, Boone (1918), registra otras tres especies: Nerocila acuminata Schioedte y Meinert, 1883; Exosphaeroma barrerae Boone, 1918 y Neotroponiscus vedadoensis (Boone, 1918). Después el mismo autor, Boone (1930; 1936), confirma la presencia en Cuba de Anilocra laticauda Milne-Edwards, 1804 y Cymothoa exigua Schioedte y Meinert, 1883 y registra por vez primera a Nerocila bartschi Boone, 1918.

Un año más tarde, aparece el trabajo de Arcangeli (1929) sobre los isópodos terrestres cubanos. En 1943, Bolívar y Peltain colectan varias especies en cuevas cubanas, pero no es hasta 1957 que Rioja publica dichos resultados (Rioja, 1957). Entre tanto, Van Name (1936) da a conocer los primeros isópodos cavernícolas cubanos, en su conocida obra sobre las especies americanas del grupo. Colman (1950), registra el isópodo gigante Bathynomus giganteus Milne-Edwards, 1879, colectado en las aguas profundas del país. Años más tarde, Holthuis y Mikulka (1972), volvieron a registrar este isópodo, para las aguas cubanas. Un poco antes, Bowman (1966), da a conocer las especies troglobias Cyathura specus y Haptolana trichostoma. 
Robert Menzies, uno de los más notables especialistas en isópodos de su época, estudiando la familia Limnoriidae, adicionó la especie Limnoria (Limnoria) tripunctata Menzies, 1951, a la lista de isópodos cubanos (Menzies, 1957). Después, en 1964, Lemos de Castro describe el isópodo terrestre cubano Trichorhina heterophthalma (Lemos de Castro, 1964).

Menzies y Glynn (1968) dieron a conocer los isópodos de Puerto Rico, mientras que Menzies y Kruczynsky (1983) publicaron sobre las especies colectadas durante los cruceros Hourglass en las aguas de Florida. Ambos trabajos resultan de enorme importancia para el estudio de los isópodos cubanos y del área. En esa época, se establece una interesante relación entre el chitón Acanthopleura granulata Meling, 1791 y el isópodo Dynamenella perforata, en Puerto Rico y Cuba (Glynn, 1968). En ella se observó que este isópodo vive introducido en el surco paleal del poliplacóforo.

Straskraba (1969), después de varios meses de trabajo en Cuba, presentó una lista de crustáceos dulceacuícolas incluyendo a Cyathura specus Bowman, 1965, además de Troglocirolana cubensis (Hay, 1903); Haptolana trichostoma Bowman, 1966 y Probopyrus panamensis Richardson, 1912 (sinónimo de $P$. pandalicola).

Los aportes más notables de todos los tiempos al conocimiento de las especies terrestres y troglobias del grupo en Cuba, fueron los realizados por Vandel (1973, 1991), al citar 63 especies de isópodos colectados por las expediciones bioespeleológicas cubano-rumanas en 1969 y 1970. Además, Coineau y Botosaneanu (1973), con material procedente de las mencionadas expediciones registran las seis primeras y únicas especies conocidas hasta el presente, de microcerbéridos cubanos.

Markham (1972, 1975 y 1985) citó varias especies de bopíridos cubanos. Ortiz et al. (1987) publicaron la "Lista de especies y bibliografía de los isópodos (Crustacea, Peracarida) de Cuba". En esta se compiló y actualizó la información sobre el grupo en Cuba (31 familias, 77 géneros y 116 especies). Los trabajos de Lalana et al. (1980), Lalana y Pérez (1985), Lalana y Ortiz(1992), Varela et al.(2003) y Lalana et al.(1985, 2005, 2007), citan los isópodos de los manglares de las lagunas costeras y playas, de la región occidental y central cubana. Después, es descrita la especie Phycolimnoria bacescui Ortiz y Lalana, 1988, actualmente Limnoria bacescui (Ortiz y Lalana, 1988).

La guía de Kensley y Schotte (1989) cita numerosas especies de isópodos presentes en las aguas cubanas. Casi una década después, Ortiz et al. (1998), describen el macho de la especie Cyathura orghidani Negoescu, 1983, mientras que García Debrás et al. (1997), actualizaron la distribución de cuatro de los isópodos troglobios cubanos.

Los trabajos de Wagelle (1982), Armas y Juarrero de Varona (1999) y de Juarrero de Varona y Armas $(1996 ; 2003)$ amplían notablemente el conocimiento de los isópodos no marinos cubanos. Los mismos autores, dan a conocer los integrantes del género Pseudarmadillo en Cuba, presentando una clave para identificar las especies cubanas y la descripción de un nuevo género y 11 especies. En ese mismo año se da a conocer la especie Brackenphilloscia vandeli proveniente de un "gour" de goteo situado en una cueva de Pinar del Río (Ortiz y Lalana, 1999). Años más tarde, como resultado del estudio de los peracáridos del blue hole de Cayo Flamenco, se describe la especie Gnathia micheli (Ortiz, et al., 2012). Mucho antes fue descrita G. hemingwayi Ortiz y Lalana (1997), colectada en la playa de Cojimar.

Finalmente, concluimos con este recuento histórico mencionando la descripción de la especie Paraimene danieli (Ortiz, et al., 2012), así como la compilación de las últimas publicaciones sobre crustáceos cubanos producidas, que incluyen varias de isópodos (Lalana et al., 2014). 


\section{Morfología}

Aunque su patrón corporal más común es semejante al de las bien conocidas "cochinillas de humedad", la morfología de los isópodos es variable. En ciertos casos, sin embargo pueden tener su cuerpo cilíndrico, como en el de los antúridos. Se diferencian de los restantes peracáridos porque presentan, casi todos, su cuerpo oval, deprimido dorso-ventralmente, respiran por sus pleópodos y poseen el primer par de pereópodos simples o subquelados (nunca quelados) y tienen el último segmento del pleón fusionado con el telson, al cual se le conoce como pleotelson. $\mathrm{Su}$ cuerpo está dividido en cabeza, pereón y pleón. Las partes corporales más importantes de estos peracáridos están señaladas a continuación (Figs. 2 y 3).
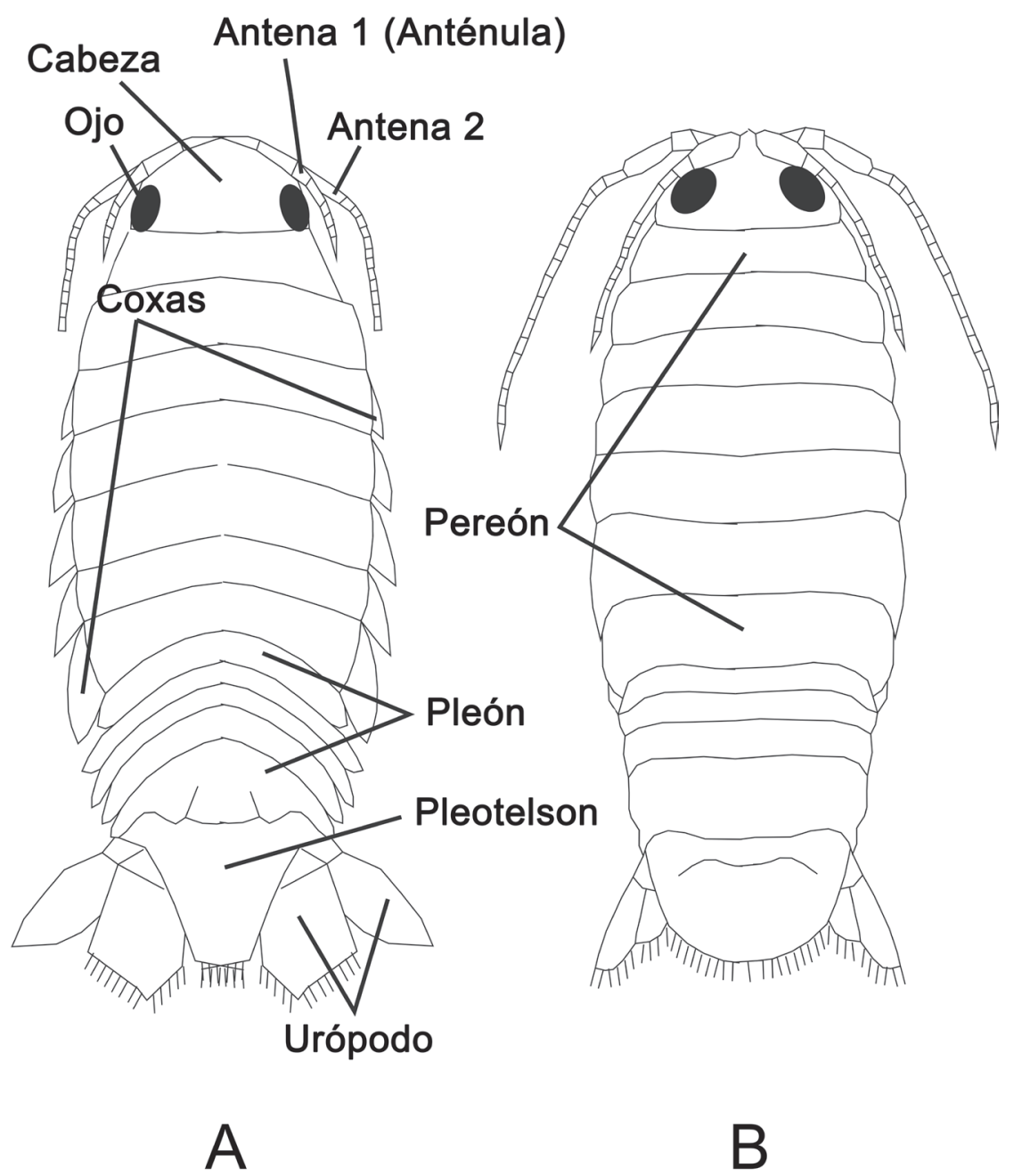

Figura 2. Vista dorsal de dos isópodos mostrando sus partes corporales. A, con coxas visibles en vista dorsal. B, con coxas no visibles en vista dorsal (tomada de Ortiz y Cházaro-Olvera, 2018). 


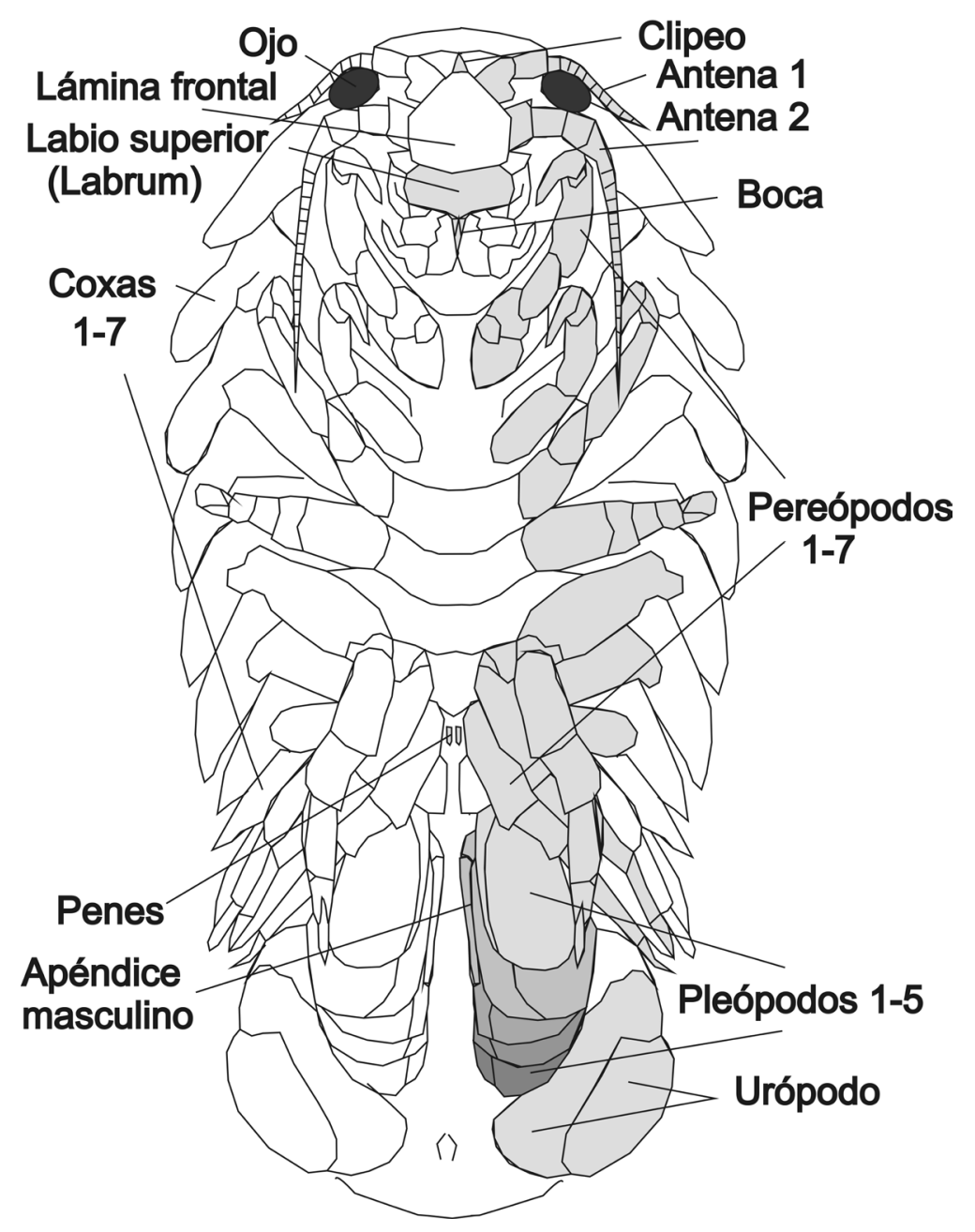

Figura 3. Vista ventral de un isópodo, mostrando sus partes corporales (tomada de Ortiz y Cházaro- Olvera, 2018).

\section{Taxonomía}

Los resultados obtenidos, después de compilar y actualizar taxonómicamente todas las especies de isópodos citadas para el país, se relacionan en las dos Tablas que se ofrecen a continuación. La Tabla I presenta las especies de isópodos marinos, estuarinos, semiterrestres y parásitos. Se ha confeccionado alfabéticamente con los datos inéditos obtenidos por los autores, durante más de 45 años de colectas en la plataforma cubana. La Tabla II se refiere a las especies terrestres y troglobias, según la provincia, localidad típica y los accidentes geográficos correspondientes. 


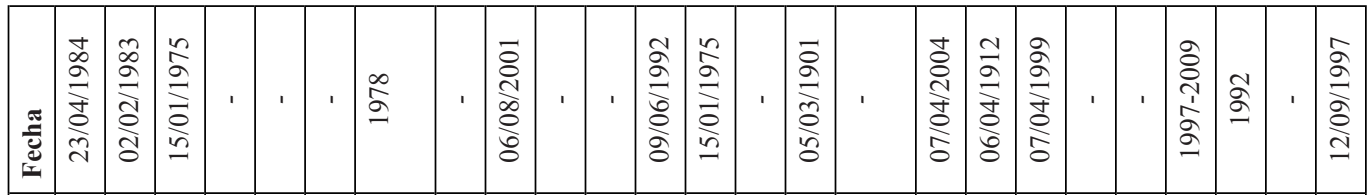

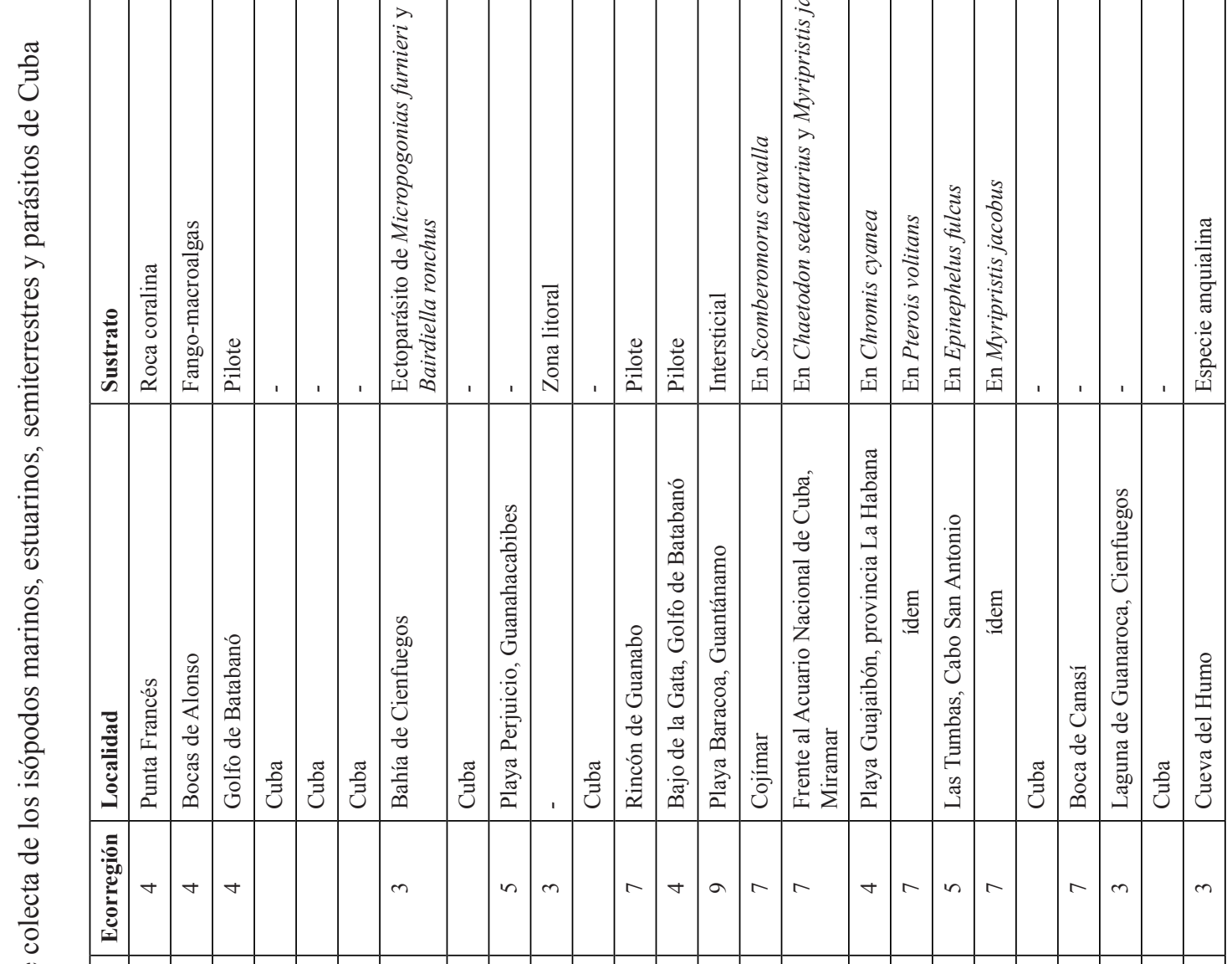

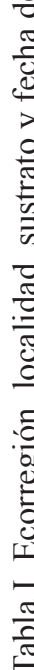

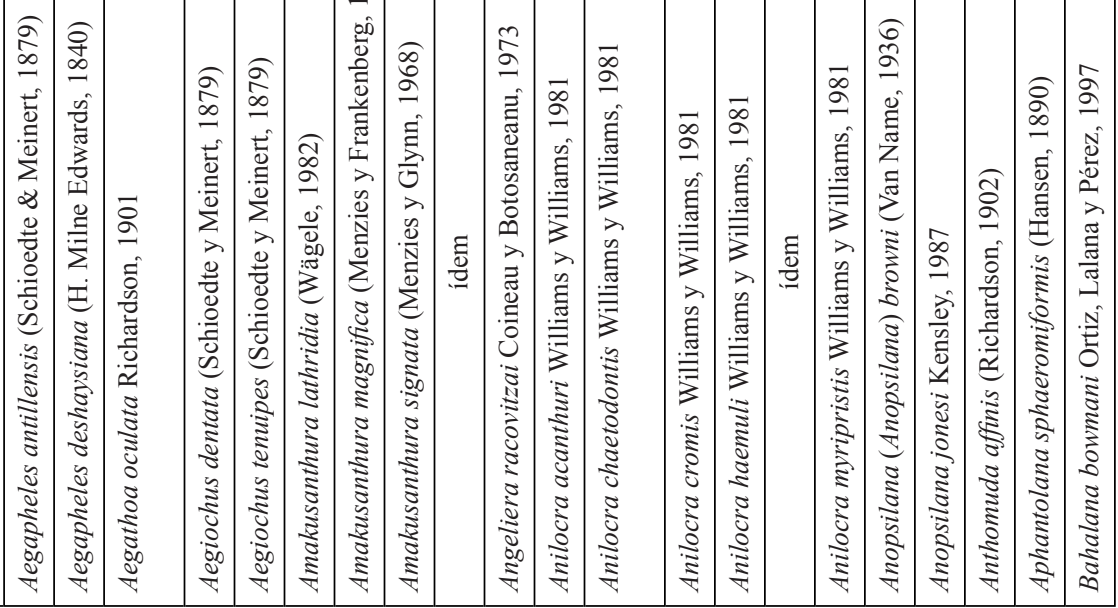




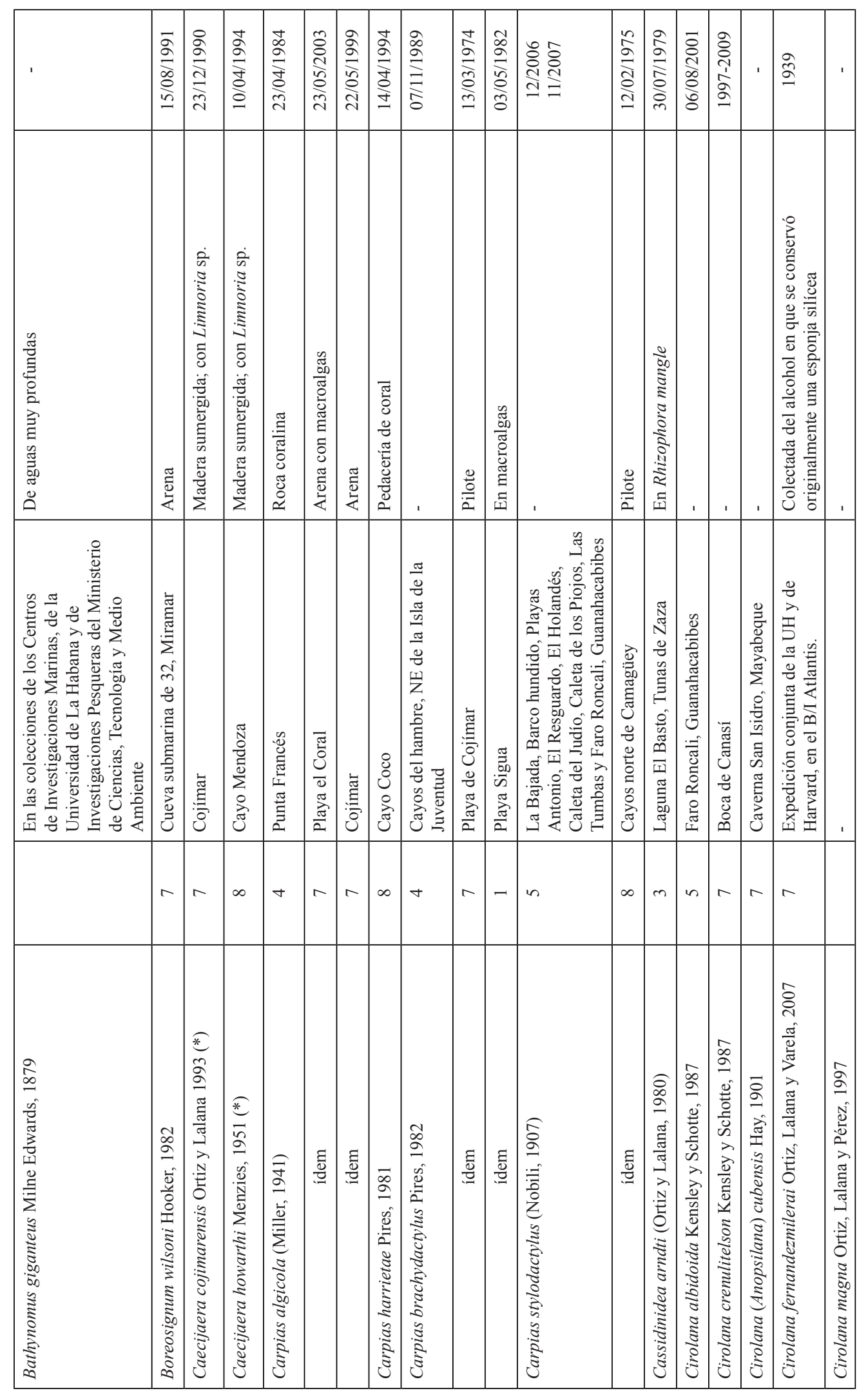




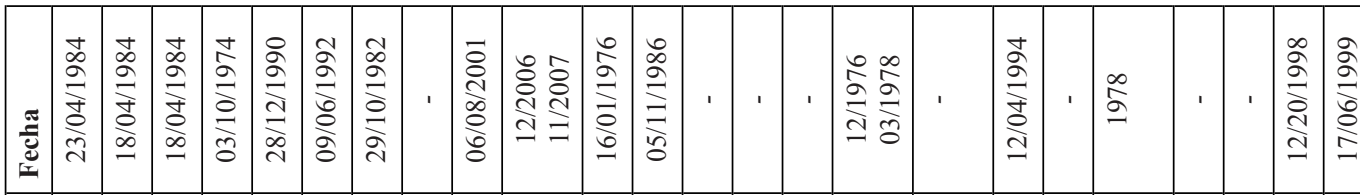

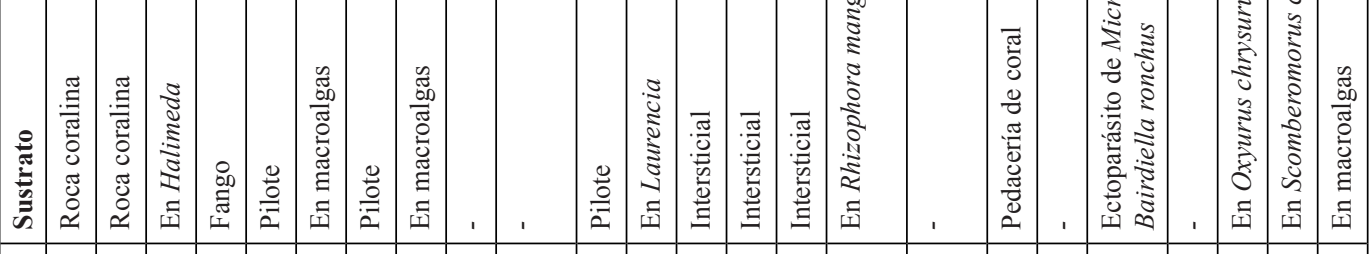

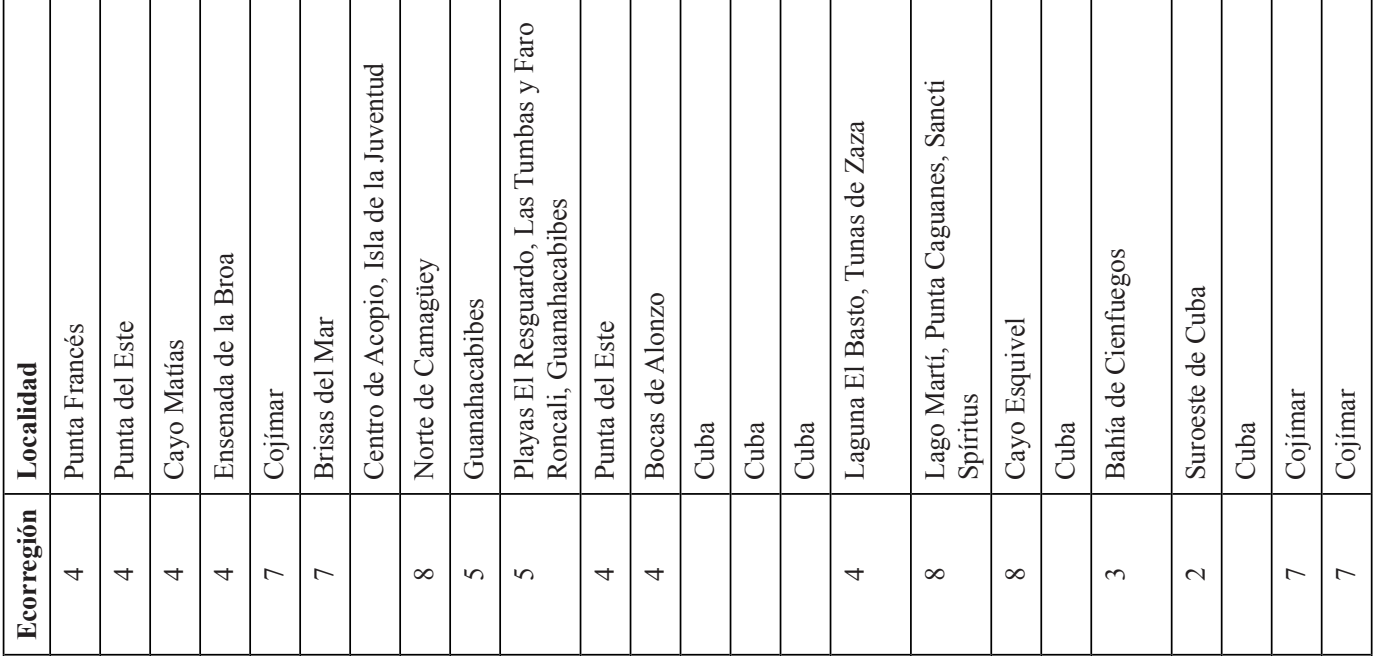

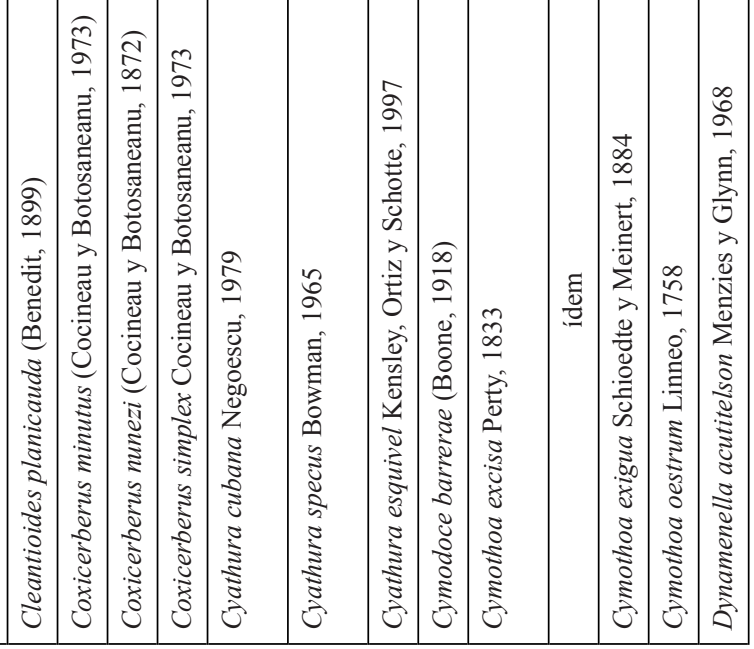




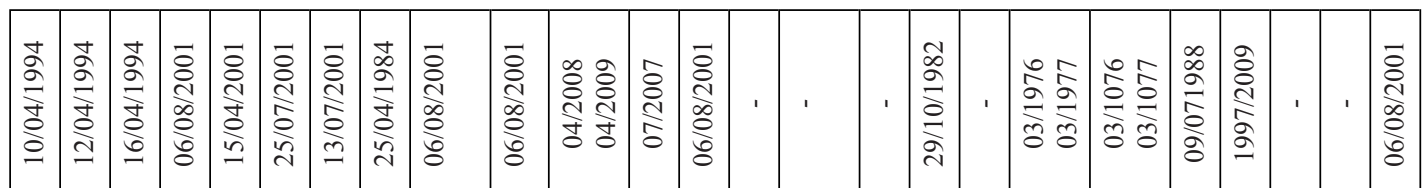

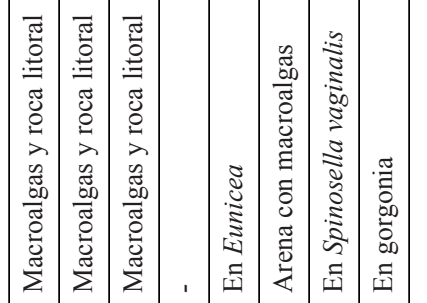

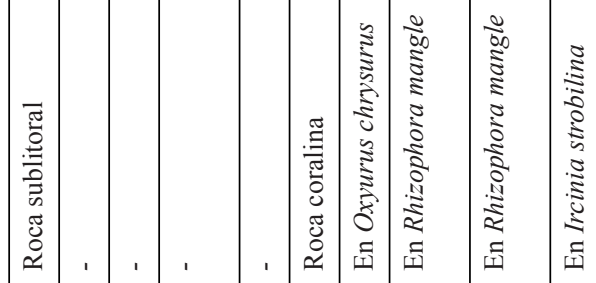

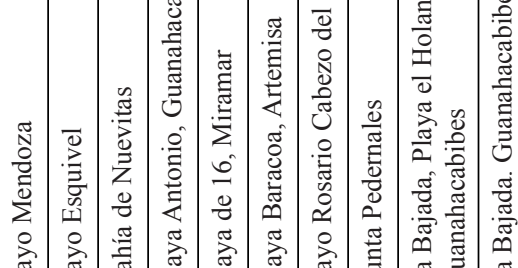

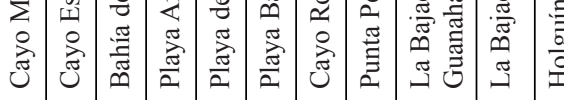

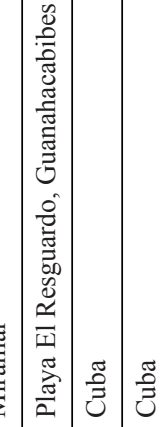

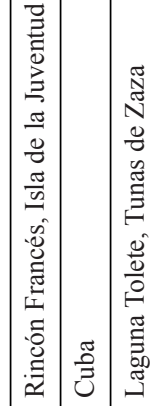

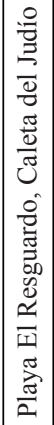

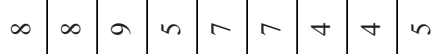



n

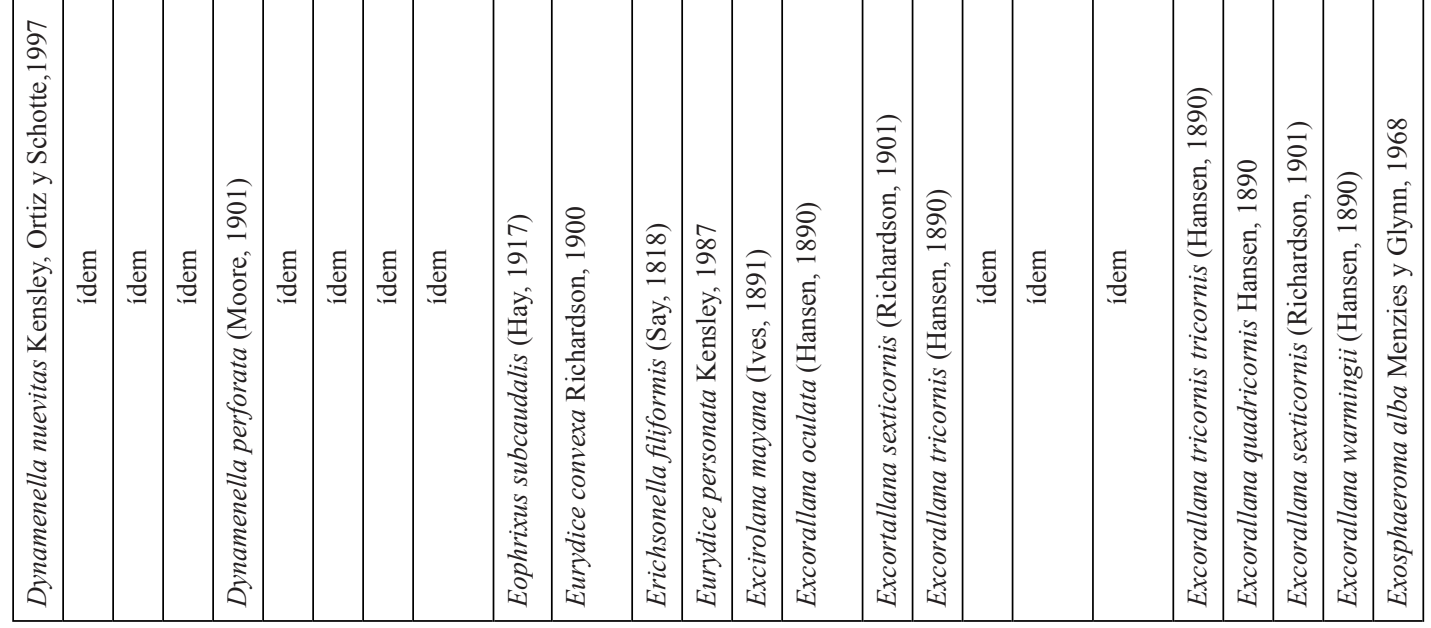




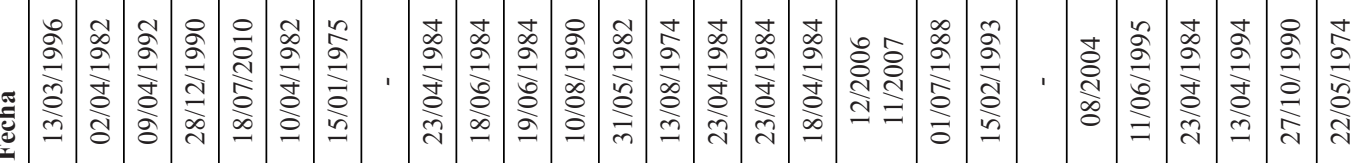

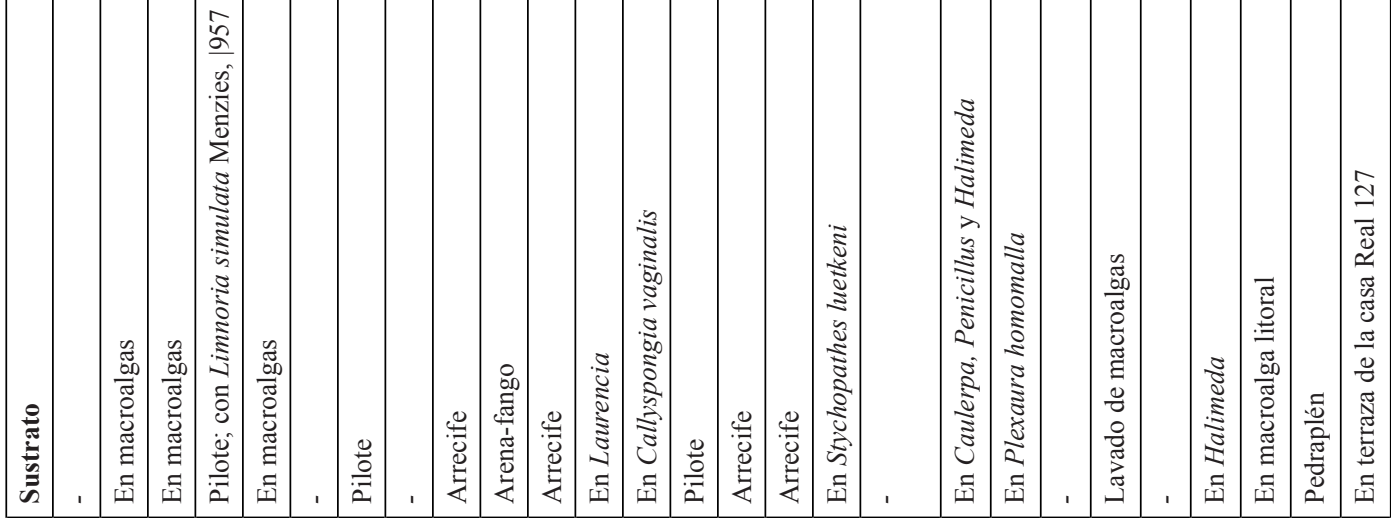

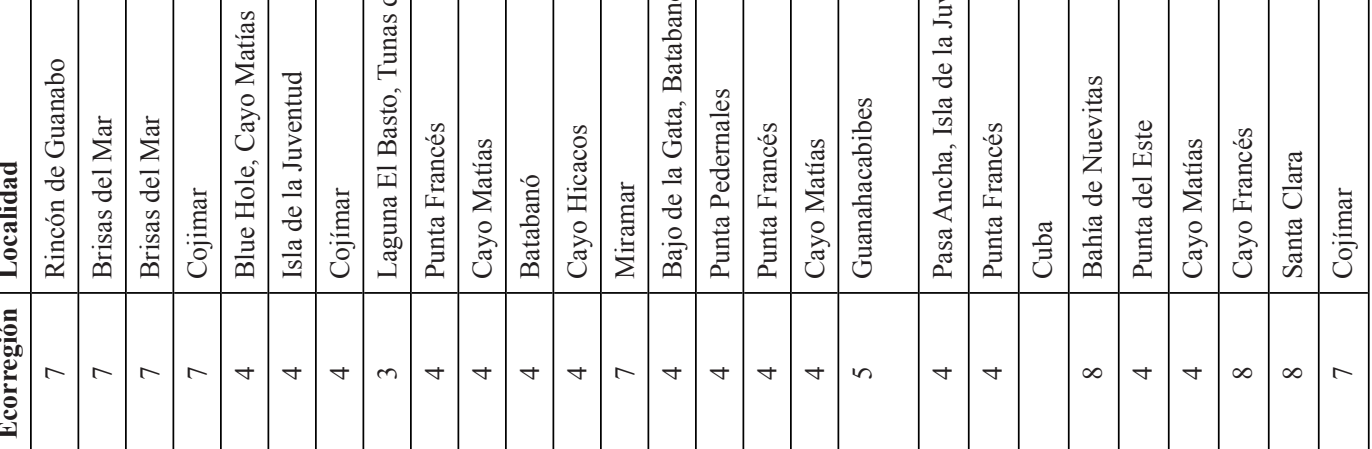




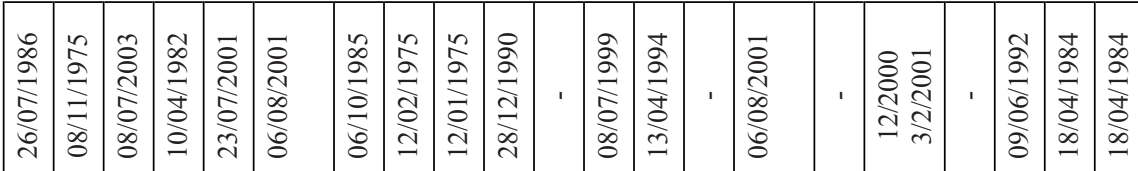

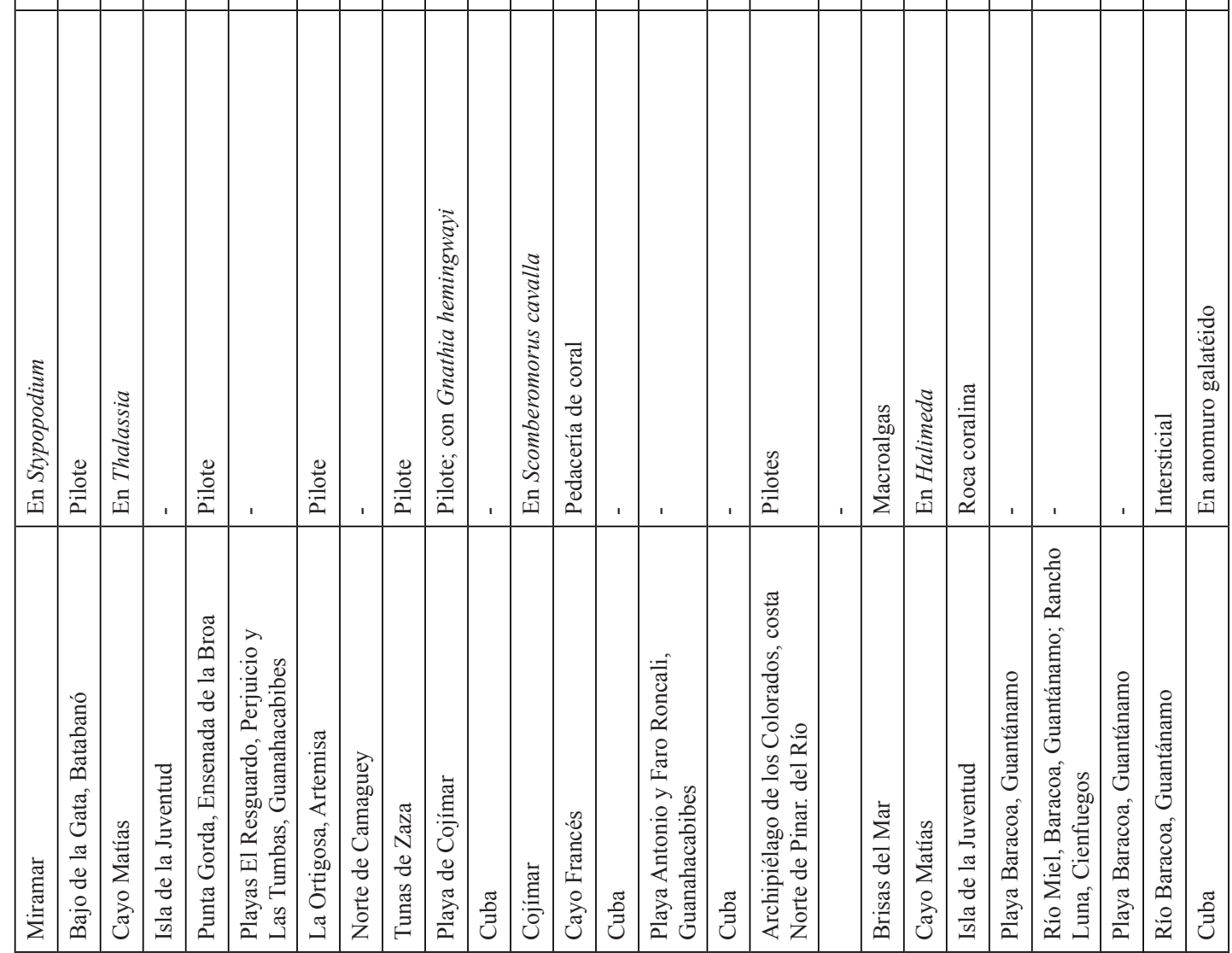

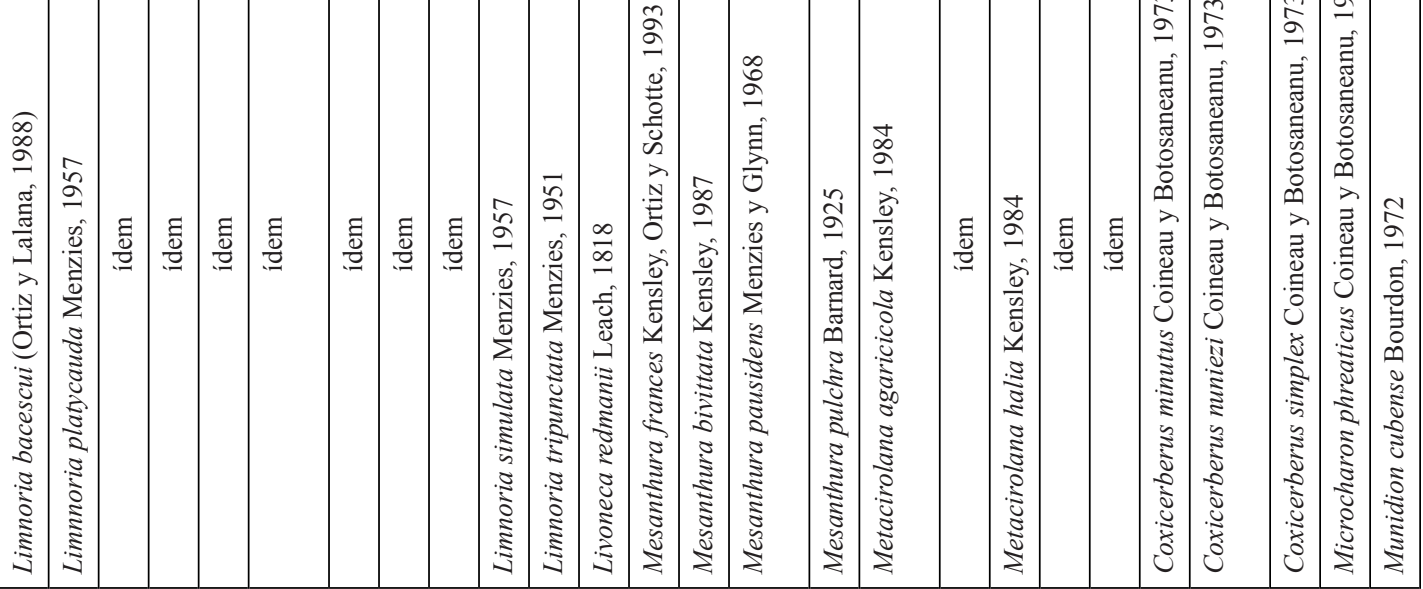




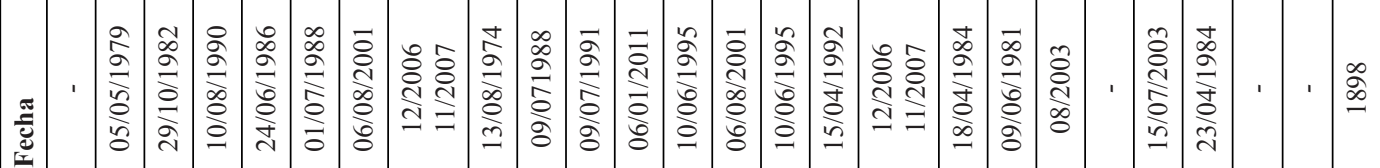

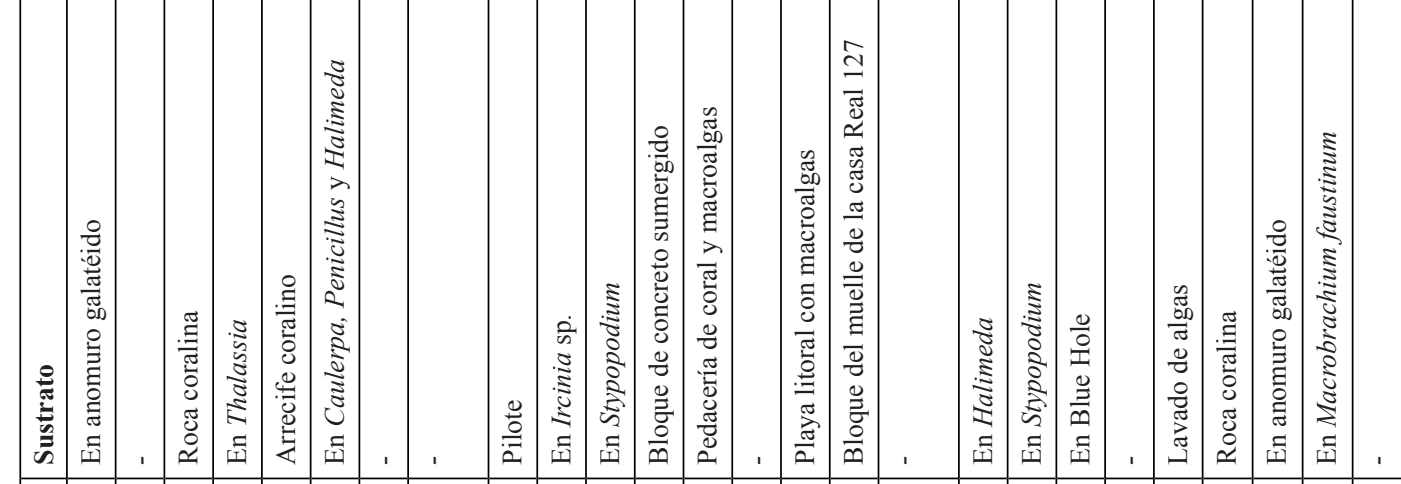

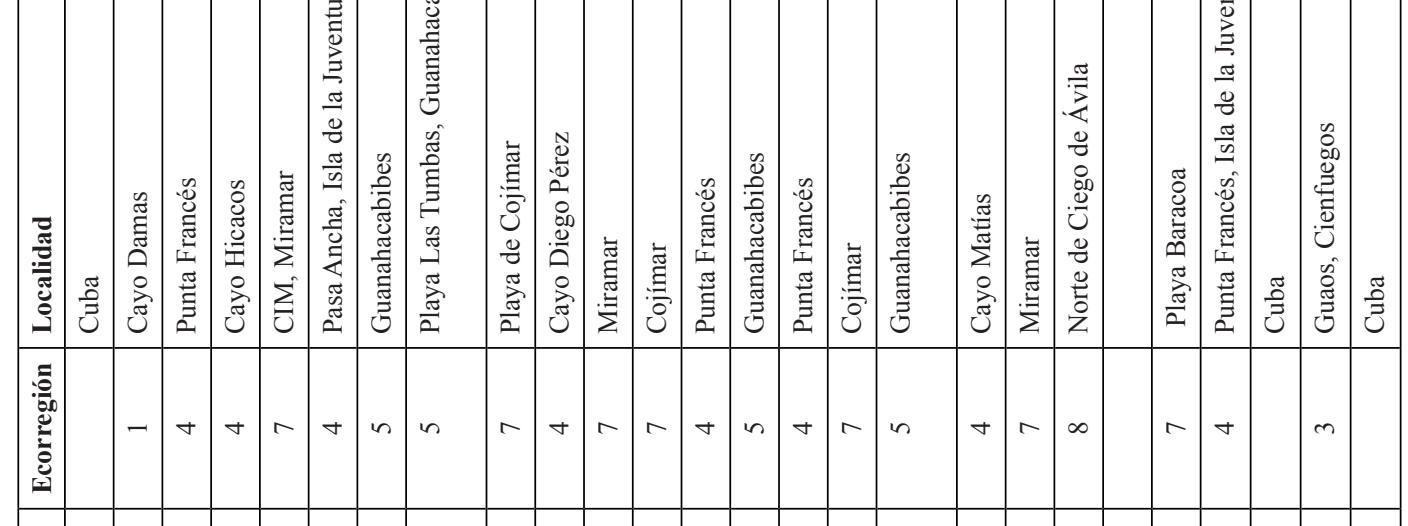

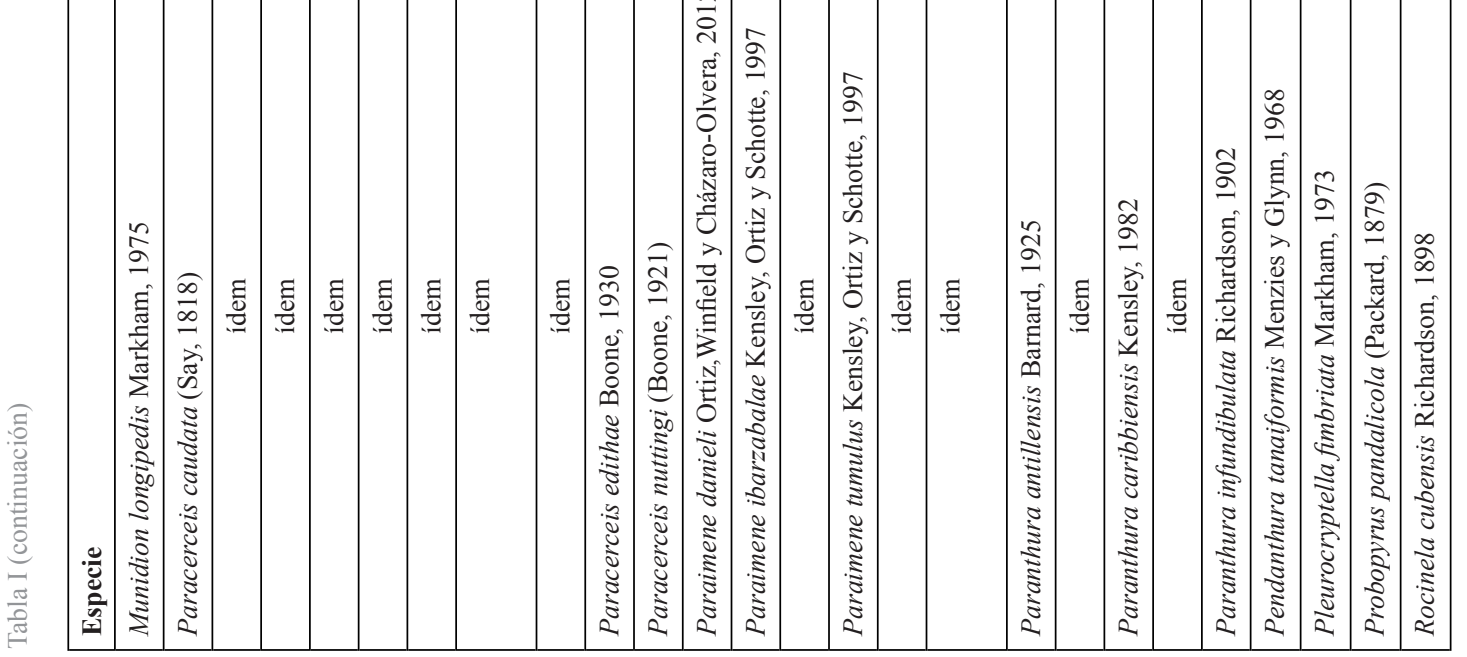




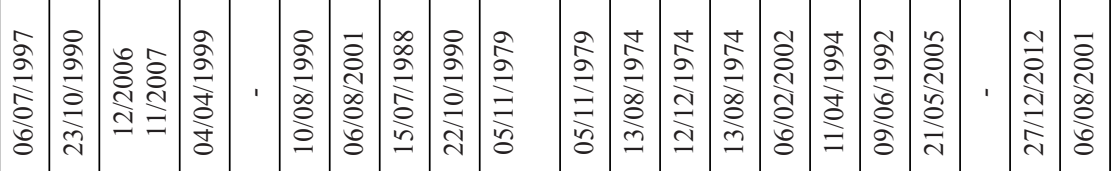

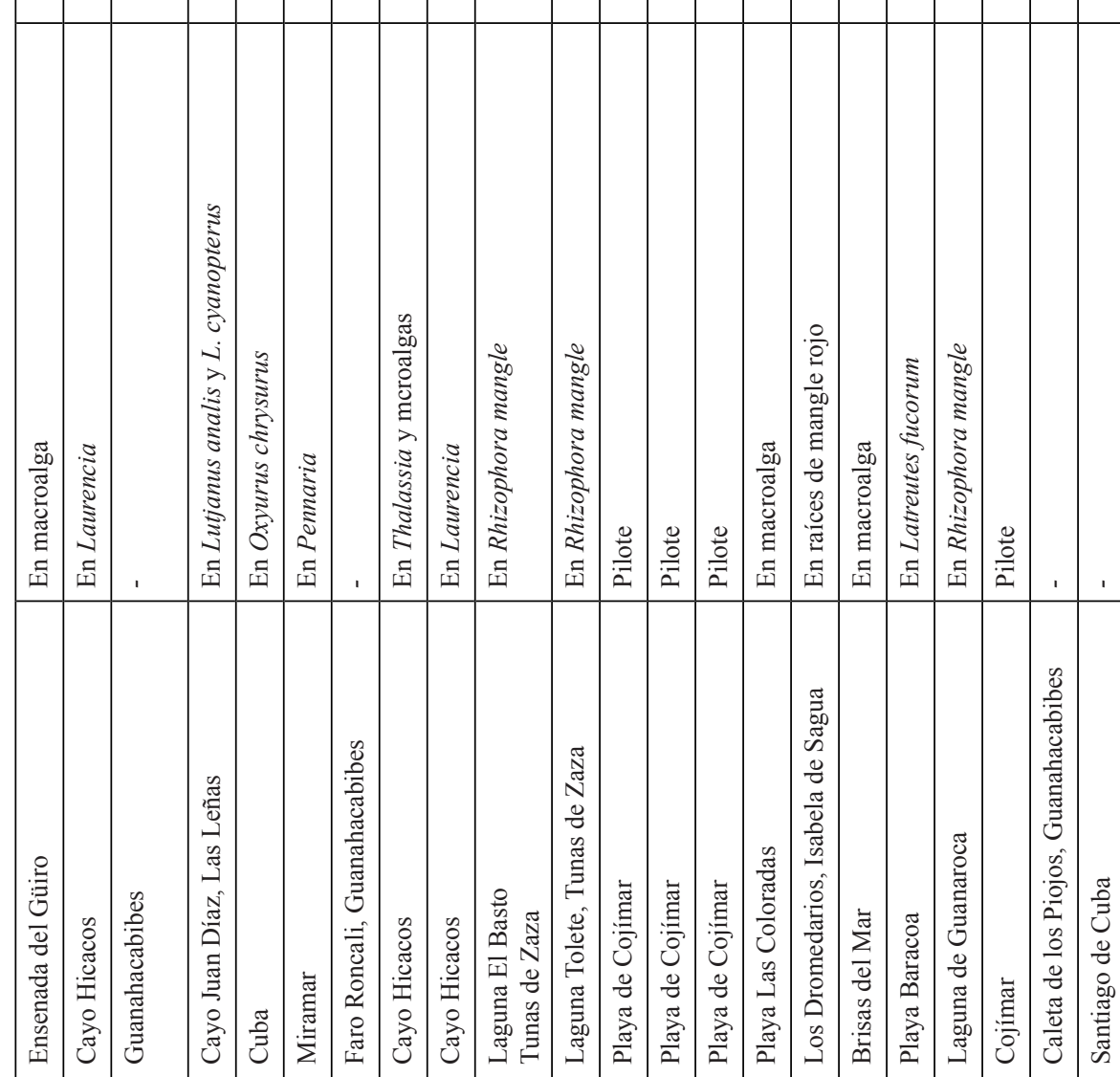

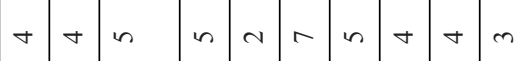

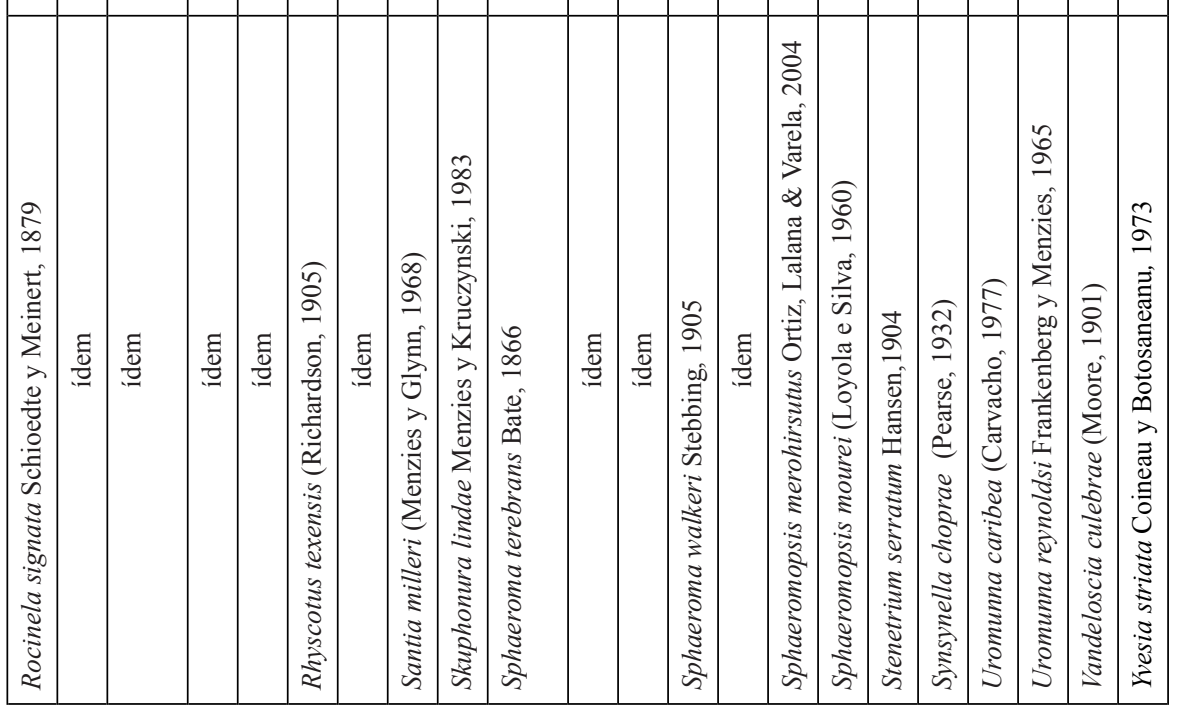

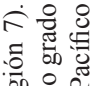

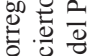

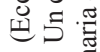

$\hat{\sigma} \dot{\bar{\sigma}} \cdot \frac{\sigma_{0}}{\bar{\sigma}}$

तf

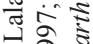

$\lambda$ 列

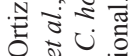

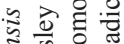

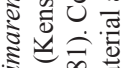

:

ن

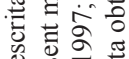

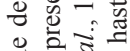

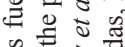

过

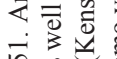

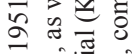

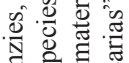

एक की

₹

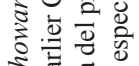

(2) 또

ธे छ है

ชूँ

记节

के 휴요율

프워

क 믈

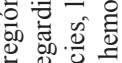

०.

过氞记

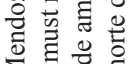

ล

至要

ธี

击

造 :

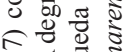

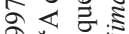

평

就造

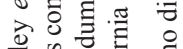

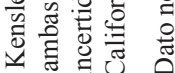


En la Tabla I se relacionan las especies marinas, estuarinas, semiterrestres y parásitas, distribuídas por ecorregiones, con sus localidades y sustratos. En ella se denota que el suborden Asellota, está relativamente bien representado en Cuba, debido a la presencia de los géneros Joeropsis (tres especies) y Carpias, Santia, Stenetrium y Uromunna, con una. Las especies Hansenium bowmani y H. spatulicarpus, son las de mayor distribución, al aparecer en cuatro localidades distintas (ecorregiones 4 y 5). Esta relación nos indica también, que los antúridos Hansenium (cuatro especies) y Accalathura y Mesantthura (con tres cada una), son los más diversos. Los aselotos más comunes en las aguas cubanas son $H$. bowmani y H. spathulicarpus.

El suborden Cymothoida, agrupa especies con una morfología muy variable. Entre ellos, los más interesantes son los antúridos. Los machos de estos isópodos son escasos, lo cual nos obliga a clasificar las hembras, por su patrón de pigmentación, que no se pierde con el paso del tiempo (Kensley y Schotte, 1989). Las especies parásitas de peces cubanos más comunes son Cymothoa, excisa, Anilocra haemuli y Rocinela signata.

Los cirolánidos, cuyo cuerpo es semejante al de las cochinillas de humedad, son muy abundantes, siendo Cirolana parva Hansen, 1890, la especie más común, presente en 10 localidades de las ecorregiones 4,5 y 8.

Un caso muy especial es el de Rocinela signata, cuya picada es semejante a la de un tábano (Tabanidae), siendo capaz de succionar sangre humana (Ortiz et al., 1996). El caso más curioso de todos los cimotóidos es el de los gnátidos (Gnathia Leach, 1814), poseen, el dimorfismo sexual más impresionante entre los isópodos de vida libre. Los machos se reconocen por el desarrollo exagerado de sus mandíbulas; las hembras, sin embargo, poseen una cabeza reducida, los segmentos pereonales aparecen abultados y translúcidos, dejando observar sus huevos. Gnathia puertoricensis está presente en las ecorregiones 3 y 4.

Se puede apreciar también que los coralánidos Excorallana tricornis y E. quadricornis son relativamente abundantes. Paracerceis caudata, entre los esferomatoideos.

Las especies perforadoras de madera en Cuba, pertenecen a los géneros Sphaeroma Latreille, 1802 (Suborden Sphaeromatidea) y Limnoria Leach, 1814 (Suborden Limnoridia). Los efectos destructivos de dichos isópodos en el manglar, los muelles con pilotes de madera y los cascos de las embarcaciones, son muy intensos (Kensle y Schotte, 1989).

Además, las especies parásitas de peces están representadas por los géneros Aegathoa, Aegapheles, Anilocra y Cymothoa. Poseen siete o cuatro pares de patas con dáctilos en forma de ganchos para fijarse en la cabeza, la lengua o las branquias de su hospedero o la base de las aletas de su hospedero. Las hembras parásitas de otros crustáceos, sin embargo, suelen fijarse generalmente en el interior de un abultamiento del carapacho del hospedero. Este tipo de parásitos está representado hasta el presente, por los géneros Munidion y Probopyrus.

Por otra parte, la pulga trepadora Ligia baudiniana es el representante más importante del suborden Oniscidea.

Se puede concluir que, los isópodos cimotóidos marinos cubanos más comunes son; Cirolana parva, Paracerceis caudata, Limnoria platycauda, Dynamenella perforata y Rocinela signata y Sphaeroma walkeri.

Finalmente, los oniscideos relacionados con el mar son escasos. Los géneros Tylos, Rhyscotus y Erichsonella, han sido señalados en las zonas costeras cubanas. 
Tabla II. Distribución de las especies terrestres dulceacuícolas y troglobias cubanas por provincias y/o accidente geográfico.

\begin{tabular}{|c|c|}
\hline Especies & Provincias y/o accidente geográfico \\
\hline Anopsilana (Anopsilana) cubensis (Hay, 1903) & $\begin{array}{l}\text { Artemisa, La Habana, Mayabeque, Matanzas, Isla de la } \\
\text { Juventud }\end{array}$ \\
\hline Archeoscia singularis Vandel, 1981 & $\begin{array}{l}\text { Pinar del Río, Mayabeque, Cuevas de Caguanes y de los } \\
\text { Animales, Sancti Spíritus y Cueva El Rabón, Guantánamo y } \\
\text { Laguna de Baconao, Santiago de Cuba, Valle del Río Jojo, } \\
\text { Arroyo de los Guineos, hoyo de Fania Pinar del Río; Cueva } \\
\text { de las Represas, Guantánamo, Cueva de Jarrito, Matanzas y } \\
\text { Sierra de la Gran Piedra }\end{array}$ \\
\hline Baconaoscia negreai Vandel, 1981 & Laguna de Baconao, Santiago de Cuba \\
\hline Bahalana bowmani Ortiz, Lalana y Pérez, 1997 & Cueva del Humo, Playa Girón, Matanzas \\
\hline Bisilvestria marrassinii Arcangeli, 1929 & Santiago de Cuba \\
\hline Brackenphiloscia vandeli Ortiz, García Debrás y Lalana, 1999 & Gran Caverna de Fuentes, Luis Lazo, Pinar del Río \\
\hline Cirolana (Anopsilana) magna Ortiz, Lalana y Pérez, 1997. & Cueva El Brinco, Playa Girón, Matanzas \\
\hline Clavigeroniscus orghidani Vandel, 1981 & Cueva El Rabón (antigua provincia de Oriente) \\
\hline Colombophiloscia romanorum Vandel, 1981 & $\begin{array}{l}\text { Gruta Jibara, Holguín y Las Cruces (antigua provincia de } \\
\text { Oriente) }\end{array}$ \\
\hline Comeodillo decoui Vandel, 1973 & Cueva del Mudo, Mayabeque \\
\hline Cubanophiloscia briani (Arcangeli, 1929) & $\begin{array}{l}\text { Cuevas de Emilio, de Jagüey, del Agua y Oscura, Pinar del } \\
\text { Río. Cueva del Baño, de los Animales, Artemisa; Cueva de } \\
\text { La Pluma, Matanzas; Grande de Caguanes, Sancti Spíritus. } \\
\text { Cuevas del Agua y del Indio, Camagüey; Guayabal, Las Tunas }\end{array}$ \\
\hline Cubanoscia primitiva Vandel, 1981 & $\begin{array}{l}\text { Holguín, Cuevas de la Cantera, Las Golondrinas y Bariay, } \\
\text { Guantánamo }\end{array}$ \\
\hline Cubanoscia romanorum Vandel, 1981 & $\begin{array}{l}\text { Cuevas del Jaguey y de la Vela, Guantánamo; Gran Caverna } \\
\text { de Santo Tomás y Hoyo de Fania, Pinar del Rio }\end{array}$ \\
\hline Cubaris murina Brandt, 1833 & Cuevas del Agua, Isla de la Juventud y El Mudo, Mayabeque \\
\hline Cuzcodinella oryx Juarrero de Varona y de Armas, 1996 & Guantánamo \\
\hline Cylindroniscus seurati Arcangeli, 1929 & $\begin{array}{l}\text { Guayabal, Las Tunas; La Gran Piedra, Cueva de Las Represas, } \\
\text { Santiago de Cuba }\end{array}$ \\
\hline Cylisticus esterelanus Verhoeff, 1917 & Cueva Fustete, Granma \\
\hline Dubioniscus negreai Vandel, 1973 & Cueva de Pío Domingo, Pinar del Río \\
\hline Jimenezia heteroclita Vandel, 1973 & Cueva de Majana, Guantánamo \\
\hline Haptolana trichostoma Bowman, 1966 & Cueva Benita, Sierra Cubitas, Cienfuegos \\
\hline Matazonellus eglisi Juarrero de Varona y de Armas, 1996 & Santiago de Cuba \\
\hline $\begin{array}{l}\text { Matazonellus turquinensis Juarrero de Varona y de Armas, } \\
1996\end{array}$ & Pico Turquino, Santiago de Cuba \\
\hline Microcerberus minutus Coineau y Botosaneanu, 1973 & Santiago de Cuba \\
\hline
\end{tabular}




\section{Tabla II (continuación)}

\begin{tabular}{|c|c|}
\hline Especies & Provincias y/o accidente geográfico \\
\hline Microcerberus nunezi Coineau y Botosaneanu, 1973 & Guantánamo \\
\hline Microcerberus simplex Coioneau y Botosaneanu, 1973 & Santiago de Cuba \\
\hline Microphiloscia trichoniscoides Vandel, 1973 & Cueva La Colorada del Maso, Santiago de Cuba \\
\hline Nagurus cubanocolens Vandel, 1981 & Mayabeque \\
\hline Nagurus cristatus Dolffus, 1889 & $\begin{array}{l}\text { Cueva Chorrera y Valle del río Cabañas, Pinar del Río, } \\
\text { Cuevas de la Virgen, Mayabeque y del Indio, Camagüey }\end{array}$ \\
\hline Oniscus asellus Linneo, 1758 & Especie introducida; sin localidad (Vandel, 1981) \\
\hline Neostenetroides schotteae Ortiz, Lalana y Pérez, 1997 & Cueva del Humo, Playa Girón, Matanzas \\
\hline Neotroponiscus vedadoensis & La Puntilla, Vedado, La Habana \\
\hline Pacroscia decoui Vandel, 1981 & $\begin{array}{l}\text { Hoyo de Fania (antigua provincia de Oriente) y Colorada de } \\
\text { Maso, Santiago de Cuba (?) }\end{array}$ \\
\hline Pacroscia elongata Vandel, 1981 & Matanzas \\
\hline Parapacroscia negreai Vandel, 1981 & Río Yumurí, Guantánamo \\
\hline Plumasicola orghidani Vandel, 1981 & Cueva La pluma, Matanzas \\
\hline Porcellio laevis Latreille, 1804 & $\begin{array}{l}\text { San Antonio de los Baños, Artemisa; Sierra de Najasa, } \\
\text { Camagüey }\end{array}$ \\
\hline Porcellionides bermudezi Boone, 1934 & Rincón de Guanabo, La Habana \\
\hline Porcellionides pruinosus (Brandt, 1833) & Cuevas del Indio, Pinar del Río y de la Virgen, La Habana \\
\hline $\begin{array}{l}\text { Pseudarmadillo agramontino Juarrero de Varona } \\
\text { y de Armas, } 1999\end{array}$ & Camagüey \\
\hline Pseuadrmadillo assoi Juarrero de Varona y de Armas, 1999 & Cienfuegos \\
\hline Pseudarmadillo auritus Juarrero de Varona y de Armas, 1999 & Sancti Spíritus \\
\hline $\begin{array}{l}\text { Pseudarmadillo bidentatus Juarrero de Varona y de Armas, } \\
1999\end{array}$ & Guantánamo \\
\hline Pseubdarmadillo buscki Boone, 1934 & Valle de Mercedes, Caimito, Artemisa \\
\hline Pseudarmadillo carinulatus Saussure, 1857 & Distribuido por todo el país \\
\hline Pseudarmadillo elegans Juarrero de Varona y de Armas, 1999 & Isla de la Juventud \\
\hline Pseudarmadillo gillianus Richardson, 1902 & $\begin{array}{l}\text { Cueva Oscura, Pinar del Río; Sierra Anafe, Artemisa, } \\
\text { Isla de la Juventud y Sierra de Cubitas, Camagüey }\end{array}$ \\
\hline $\begin{array}{l}\text { Pseudarmadillo holguinensis Juarrero de Varona y de Armas, } \\
1999\end{array}$ & Holguín \\
\hline Psdeudarmadillo hoplites Boone, 1934 & Sierra de Cubitas y de Najasa, Camagüey \\
\hline Pseudarmadillo jaumei Juarrero de Varona y de Armas, 1999 & Guantánamo \\
\hline Pseudarmadillo maiteae Juarrero, 2002 & Santiago de Cuba \\
\hline Pseudarmadillo mitratus Juarrero de Varona y de Armas, 1999 & Las Tunas \\
\hline Pseudarmadillo nanus Juarrero de Varona y de Armas, 1999 & Cienfuegos \\
\hline Pseudarmadillo spinosus Juarrero de Varona y de Armas, 1999 & Sancti Spíritus \\
\hline $\begin{array}{l}\text { Pseudarmadillo vansicklei Juarrero de Varona } \\
\text { y de Armas, } 1999\end{array}$ & Santiago de Cuba \\
\hline Puteosia silvestrii Vandel, 1981 & Arroyo Pozo Azul, Guantánamo \\
\hline
\end{tabular}




\begin{tabular}{|c|c|}
\hline Especies & Provincias y/o accidente geográfico \\
\hline Rabdoniscus robustus Vandel, 1981 & Las Tunas y Granma \\
\hline Rhyscotoides cubensis (Bude Lund, 1908) & Cuba \\
\hline Scleropactes botosaneanui Vandel, 1973 & Cueva de Bellamar, Matanzas \\
\hline Stygocyathura cuborientalis (Botosaneanu y Stock, 1982) & Cuba \\
\hline Stygocyathura orghidani Negoescu Vladescu, 1983 & Pinar del Río \\
\hline Stygocyathura specus Bowman, 1965 & Ciego de Ávila \\
\hline Styloniscus romanorum Vandel. 1973 & $\begin{array}{l}\text { Cuevas del Tunel, Pinar del Río, Grande, Sancti Spíritus; } \\
\text { Pío Domingo y del Salón, Pinar del Río }\end{array}$ \\
\hline Styloniscus simplex Vandel, 1981 & Las Tunas \\
\hline Sulesoscia epigea Vandel, 1973 & Gran Caverna de Santo Tomás, Pinar del Río. \\
\hline Thomasoniscus angulatus Vandel. 1981 & Pinar del Río, Mayabeque \\
\hline Trichorhina bequaerti Van Name, 1936 & $\begin{array}{l}\text { Cuevas de Jagüey, Gran Caverna de Santo Tomás y del Agua, } \\
\text { Guanahacabibes, Pinar del Río; Parque Lenin, } \\
\text { La Habana; Cueva Aguas Gordas, Holguín }\end{array}$ \\
\hline Trichorhina gianelli Arcangeli, 1929 & Pinar del Río, Santiago de Cuba, Holguín \\
\hline Trichorhina heterophthalma Lemos de Castro, 1964 & $\begin{array}{l}\text { Cuevas Grande, Yaguajay, Sancti Spíritus; La Patana, Maisí, } \\
\text { Guantánamo: Las Rosas, Banes, Holguín; del Fustete, } \\
\text { Granma; del Mudo, Catalina de Guines, Mayabeque; de La } \\
\text { Pluma; Bacunayagua, de Los Lagos o del Agua, Matanzas; } \\
\text { Bahía La Gloria Camaguey; Río Baracoa, Guantánamo }\end{array}$ \\
\hline Trichorhina tomentosa Bude Lund, 1893 & Cueva del Cura, Jaruco, Mayabeque \\
\hline Trichoniscus pseudopusillus Arcangeli, 1929 & Pinar del Río, Santiago de Cuba \\
\hline Trichoniscus pusillus Brand, 1833 & Santiago de Cuba \\
\hline Troglopiloscia silvestri Brian, 1929 & $\begin{array}{l}\text { Cuevas del Cura, Mayabeque; de Bellamar y de La Pluma, } \\
\text { Matanzas }\end{array}$ \\
\hline Tylos niveus Budde Lund, 1885 & La Puntilla, Cojímar; La Habana \\
\hline Thomasoniscus angulatus Vandel, 1981 & Santiago de Las Vegas, La Habana \\
\hline Venezillo colomboi (Arcangeli, 1929) & $\begin{array}{l}\text { Santiago de Las Vegas, La Habana; Cueva del Mudo, } \\
\text { Artemisa y Río San Vicente, Pinar del Río }\end{array}$ \\
\hline Venezillo grenadensis (Budde-Lund, 1893) & Santiago de las Vegas, La Habana \\
\hline Venezillo ramsdeni (Boone, 1934) & Santiago de Cuba \\
\hline Venezillo sanchezi (Boone, 1934) & Río Almendares, La Habana \\
\hline Venezillo silvarum (Dolffus, 1896) & Cuba \\
\hline Yvesia striata Coineau y Botosaneanu, 1973 & Santiago de Cuba \\
\hline
\end{tabular}

Los isópodos terrestres cubanos se conocían relativamente bien, gracias a citas esporádicas realizadas en trabajos de autores extranjeros. Sin embargo, luego de la realización de las expediciones cubano-rumanas y de las publicaciones de Vandel (1973, 1991), el avance sobre el conocimiento de estos peracáridos terretres e hipogeos, ha sido importante. Después, el grupo ha recibido muy poca atención. La Tabla II muestra los avances taxonómicos publicados por el mencionado especialista, junto a otros más recientes de carcinólogos cubanos (Vandel, 1973, 1991; Juarrero de Varona y Armas, 1996, 2003; Armas y Juarreo de Varona, 1999; Ortiz y Lalana, 1999). 


\section{CONCLUSIONES}

En Cuba se conocen 106 géneros y 194 especies de isópodos, de los cuales 57 géneros y 118 especies son marinos, estuarinos, semiterrestres y parásitos, mientras que 47 géneros y 76 especies son terrestres o troglobias.

Después de la publicación de Ortiz et al. (1987), los mayores avances en el estudio de las especies marinas y estuarinas en Cuba, se deben principalmente a los trabajos llevados cabo por los mismos autores y por Kensley et al. (1997). Si el número de especies marinas, estuarinas y parásitas cubanas se comparan el de las especies citadas por Kensley y Schotte (1989) y por Schotte et al. (2009), se observa que el grupo en Cuba, no ha sido estudiado con la intensidad necesaria.

Se denota la falta de información sobre las especies parásitas. Debe suponerse que en el trópico, donde se sitúa el archipiélago cubano, debería haber una mayor incidencia de registros de este tipo. El trabajo de Kensley y Schotte (1989) cita más de 50 isópodos parásitos en la región.

Es indudable que el mayor esfuerzo taxonómico entre los isópodos cubanos marinos y estuarinos se ha desarrollado en la región occidental, sobre todo en las costas cercanas a La Habana y en el golfo de Batabanó. De la ecorregión 9 prácticamente no hay información.

Los mayores aportes al estudio de las especies terrestres y troglobias cubanas se deben a las colectas de isópodos realizadas durante el desarrollo de las expediciones cubano-rumanas, que fueron publicados por Vandel $(1973 ; 1991)$. Antes se conocía muy poco de este tipo de isópodos en el país. Se estima que falta mucho por conocer sobre las especies terrestres cubanas.

\section{AGRADECIMIENTOS}

Se agradece la colaboración de Brian Kensley y Marilyn Schotte, entonces del laboratorio de crustáceos de Smithsonian Institution, durante las expediciones Cuba-USA, desarrolladas en las ecorregiones 8 y 4, a bordo del B/I "Ulises", en el año1994, y del B/I “Felipe Poey", en 1995. También a todos los biólogos, pescadores, tripulantes y alumnos, que han apoyado nuestro trabajo de tantos años.

\section{LITERATURA CITADA}

Ahyong, S. T., J. K. Lowry, M. Alonso, R. N. Bamber, G. A. Boxshall, P. Castro, S. Gerken, G. S. Karaman, J. W. Goy, D. S. Jones, K. Meland, D. C. Rogers y J. Svavarsson. 2011. Subphylum Crustacea, Brunnich, 1772. En Z-Q Zhang (Editor) Animal biodiversity: An outline of higher-level classification and survey of taxonomic richnes. Zootaxa, 3148: 165-191.

Arcangeli, A. 1929, Isopodi terrestre racoltii in Cuba da Prof. Silvestri. Bull. Lab. Zool. Pontici, 23: $130-148$.

Areces, A. 2012. Taller de regionalización y clasificación de hábitats marinos en la plataforma cubana. Instituto de Oceanología, La Habana, Cuba.

Armas, L. de y A. Juarrero de Varona. 1999. Sistemática de la familia Delatorreidae. Avicennia, 10-11: 1-42. 
Boone, P. L. 1918. Description of ten new isopods. Proc. U. S. Nat. Mus., 54: 591-604.

Boone, P. L. 1930. Crustacea: Anomura, Macrura, Schizopoda, Isopoda, Amphipoda, Mysidacea, Cirripedia and Copepoda. Scientific results of the cruises of the Yachts "Eagle" and "Ara" 1921-1928, W. K. Vanderbilt, Commanding in Bull. Vanderbilt Marine Museum 3: 1-118.

Boone, P. L. 1936. Crustacea, Anomura, Macrura, Schyzopoda, Isopoda, Amphipoda, Mysidacea, Cirripedia and Copepoda. Scientific results of the cruises of the yacths Eagle and Ara 19211928 Wlliam T. Vanderbilt Commanding. Bulletin of the Vanderbilt Marine Museum, 31: 1-118.

Bowman, T. E. 1965. Cyathura specus a new cave isopod from Cuba. Studies on the fauna of Curaçao and other Caribbean islands, 72: 78-97.

Bowman, T. E. 1966. Haptolana trichostoma a new genus and species of troglobitic cirolanid isopod from Cuba. International Journal of Speleology, 2: 107-108.

Boyko, C. B, N. L. Bruce, K. A. Hadfield, K. L. Merrin, Y. Ota, G. C. B. Poore, S.Taiti, M. Schotte y G. D. F. Wilson (Eds). (2008 onwards). World Marine, Freshwater and Terrestrial Isopod Crustaceans database. Accessed at http://www.marinespecies.org/ isopoda on 2018-05-18.

Coineau, N. y L. Botosaneanu. 1973. Isopodes interstitieles de Cuba. Resultats des Expeditions Biospeologiques Cubano-Rumanies a Cuba. Edit. Acad. R. S. Romania 1: 424 pp.

Colman, J. S. (1950). The Sea and Its Mysteries, G. Bell and sons, London, 285 pp.

García Debrás, A., A. Pérez González, y M. Ortiz. 1997. Distribución geográfica de los crustáceos peracáridos acuáticos (Mysidacea, Amphipoda, Isópoda), de las cuevas de Cuba. Cocuyo, 6: 33-36.

Glynn, P. W. 1968. Ecological studies on the associations of chitons in Puerto Rico with special rewference to Spaeromid isopods. Bulletin of Marine Science, 16 (3): 572-626.

Holthuis. L. B. y W. R. Mikulka. 1972. Notes on the deep-sea isopods of the genus Bathynomus A. Milne Edwards, 1979. Biological Results of the University of Miami Deep-sea Expeditions 91. Bulletin of Marine Science, 22 (3): 575-591.

Juarrero de Varona, A. y L. de Armas. 1996. Nuevo género de isópodo terrestre (Isopoda, Armadillidae) de Cuba. Avicennia, 4: 95-102.

Juarrero de Varona, A. y L. de Armas. 2003. A new species of terrestrial isopod (Oniscidea, Delatorreidae) Avicennia, 16: 97-102.

Kensley, B. y M. Schotte. 1989. Guide to the marine isopod crustaceans of the Caribbean. Smithsonian Institution Press, Washington D.C. pp. 308.

Kensley, B., M. Ortiz y M. Schotte. 1997. New records of marine Isopods from Cuba (Crustacea, Peracarida). Proccedings of the Biological Society of Washington, 110 (1): 74-98.

Lalana, R., M. Ortiz y O. Gómez. 1980. Lista de invertebrados bentónicos de las lagunas costeras Tolete y El Basto de la zona de Tunas de Zaza. Revista de Investigaciones Marinas, 1 (1): 19-45. 
Lalana, R., M. Álvarez, M. Ortiz, R. Pérez y T. Veledo. 1985. Organismos asociados a las raíces de mangle Rhizophora mangle, en lagunas costeras y cayos. Revista de Investigaciones Marinas, 6 (2-3): 59-71.

Lalana, R. y M. Pérez-Moreno. 1985. Estudio cualitativo y cuantitativo de la fauna asociada a las raíces de Rhozophora mangle en la cayería Este de la Isla de la Juventud. Revista de Investigaciones Marinas, 6 (2-3): 45-58.

Lalana R. y M. Ortiz. 1992. Fauna asociada a mangles de la Laguna Guanaroca, Provincia de Cienfuegos, Cuba. Revista de Investigaciones Marinas, 13 (3): 205-214.

Lalana, R., M. Ortiz y C. Varela. 2005. Primera adición a la lista de los crustáceos no decápodos de Cuba. Revista Biología, 19 (1-2): 50-56.

Lalana, R., M. Ortiz y C. Varela. 2007. Crustáceos (Arthropoda: Crustacea) de la playa María la Gorda, costa sur de la península de Guanahacabibes, Pinar del Río, Cuba. Revista Biología, Vol. 21, No. 1-2: 1-8.

Lalana, R., M. Ortiz y C. Varela. 2014. Segunda adición a la lista de los crustáceos (Arthropoda: Crustacea) de aguas cubanas. Revista de Investigaciones Marinas, 34 (1): 121-131.

Markhan, J. C. 1973. Six new species of bopyrid isopopds parasitic on Galatheid Crabs of the genus Munida in the Western Atlantic. Bulletin of Marine Science, 23 (3): 613-648.

Markhan, J. C. 1975. A review of the genus Munidion. Hansen, 1877, parasitic on Galatheid Crabs in the Atlantic and Pacific Oceans. Bulletin of Marine Science, 25 (3): 422-441.

Markhan, J. C. 1985. A review of the bopyrid isopods infesting caridean shrimps in the Northwestern Atlantic Ocean with special reference to those collected during the Hourglass Cruises in the Gulf of Mexico. Memories of the Hourglass Cruises, 7 (3): 1-156.

Menzies, R. 1957. The Marine family Limnoridae (Crustacea. Isopoda) Part. 1. Northern and Central America Systematic, Distribution and Ecology. Bulletin of Marine Science Gulf Caribbean, 7 (2): 101-200.

Menzies, R. y P. Glynn. 1968. The common marine isopod Crustacea of Puerto Rico. Studies Fauna Curacao and other Caribbian Islands, 27: 1-33.

Menzies, R. J. y W. Kruczynsky. 1983. Memoirs of the Hourglass Cruises - Isopoda crustacea (Exclusive of Epicaridea). Florida Departament of Natural Resources. Florida, Vol. 6. $125 \mathrm{pp}$.

Ortiz, M., R. Lalana y O. Gómez, 1987. Lista de especies y bibliografía de los isópodos (Crustacea, Peracarida) de Cuba. Revista de Investigaciones Marinas, 8 (3): 29-37.

Ortiz, M. y R. Lalana, 1988. Una nueva especie de isópodo del género Phycolimnoria (Isopoda, Limnoridae), de aguas cubanas. Revista de Investigaciones Marinas, 9 (2): 37-42.

Ortiz, M., R. Lalana y O. Gómez. 1996. Registros de picadas al hombre de Rocinela signata (Crustacea, Isopoda, Aegidae), en Cuba. Revista de Investigaciones Marinas, 14 (2-3): 167-168. 
Ortiz, M. y R. Lalana. 1997. Gnathia hemingwayi especie nueva (Isopoda, Gnathidea) de la costa noroccidental de Cuba. Revista de Investigaciones Marinas, 18 (1): 21-26.

Ortiz, M., R. Lalana y A. Pérez. 1997. Tres nuevas especies de isópodos cavernícolas (Crustacea) de la Isla de Cuba. Revista de Investigaciones Marinas, 18 (1): 1-20.

Ortiz, M., A. García-Debrás y R. Lalana, 1998. Nueva localidad y descripción del macho de Cyathura orghidani Negoescu Vladescu. Revista de Investigaciones Marinas, (20) 2-3: 106-107.

Ortiz, M. y R. Lalana. 1999. Un nuevo género y una nueva especie de isópodo cavernícola (Isopoda, Oniscidea), de la Isla de Cuba. Revista Investigaciones Marinas, 20 (1-3): 108-112.

Ortiz, M., R. Lalana y C. Varela. 2012. First record of peracarid crustaceans from the Cayo Matías Blue Hole SW Cuba with the description of two new species. Zootaxa, 3505: 55-66.

Ortiz, M., I. Winfield y S. Cházaro-Olvera. 2012. A new species of isopoda (Isopoda, Flabellifera; Sphaeromatidae) from Cuba, with an identification key for the species of Paraimene. Revista Mexicana de Biodiversidad, 83: 976-982.

Ortiz, M. y S. Cházaro-Olvera. 2017. Isópodos marinos (Crustacea: Peracarida) de las aguas someras mexicanas del Golfo de México (excluyendo Epicaridea). Manual de identificación. Universidad Nacional Autónoma de México, Facultad de Estudios Superiores Iztacala, $91 \mathrm{pp}$.

Richardson, H. 1901. Key to the isopods of the Atlantic Coast of North America with descriptions and new and little known species. United States National Museum, Procceding, 23: 493-579.

Richardson, H. 1905. A Monograph of the Isopods of North America. Bull. U. S. Nat. Mus., 54: 1-727.

Rioja, E. 1957. Estudios Carcinológicos. XXXV, Datos sobre algunos Isópodos cavernícolas de la Isla de Cuba. Anales del Instituto de Biología, 27.

Saussure, H. 1857. Diagnose de quelques Crustaces nouveaux des Antilles et du Mexique. Revue et Magasin de Zoologia, 9: 304-308.

Schotte, M., J. C. Markham y G. D. F. Wilson. 2009. Isopoda (Crustacea) of the Gulf of Mexico, Pp. 973-986 in Felder, D.L. and D.K. Camp (eds.), Gulf of Mexico-Origins, Waters, and Biota. Biodiversity. Texas A\&M University Press, College Station, Texas.

Straskraba, M. 1969. Lista de los crustáceos dulceacuícolas de Cuba y sus relaciones Zoogeográficas. Academia de Ciencias de Cuba. Serie Biológica, 8: 1-37.

Vandel, A. 1973. Les isopodes terrestres et cavernicoles de l'ille de Cuba. Resultats des Expeditions Biospeologiques Cubano-Rumanies a Cuba. Edit. Acad. R. S. Romania, 1: $424 \mathrm{pp}$.

Vandel, A. 1991. Les isopodes terrestres et cavernicoles de l'ille de Cuba. (2) Resultats des Expeditions Biospeologiques Cubano-Rumanies a Cuba. Editura Academie R. S. Romania, 1: $190 \mathrm{pp}$. 
Van Name, W. G. 1936. The American Land and Fresh Water Isopod Crustacea. Bulletin of the American Museum of Natural History, 71: 535 pp.

Varela, C., M. Ortiz y R. Lalana. 2003. Nota científica, Crustáceos (Peracarida y Decapoda), de la costa sur de la península de Guanahacabibes. Revista de Investigaciones Marinas, 24 (1): 72-76.

Wagelle, J. W. 1982. On Apanthuretta lathridia n. sp. (Crustacea, Isopoda, Anthuridea) from Cuba. Bijdragen tot de Dierkunde, 52 (1):41-46.

World Register of Marine Species (WoRMS). Available from ttp://www.marinespecies.org at VLIZ. Accessed 2016-10-08. doi:10.14284/170.

\section{ANEXO 1.}

\section{TRABAJOS NO CITADOS EN EL TEXTO, QUE SE REFIEREN A ISÓPODOS CUBANOS}

Alayo, O. P. 1974. Guía elemental de las aguas dulces. Torreia, nueva serie, 17: 1-79.

Barro, A., E. Fonseca, M. Ortiz y R. Lalana. 2013. Lista de los crustáceos marinos y estuarinos (Arthropoda, Crustacea) de Boca de Canasí, Mayabeque, Cuba. Revista cubana de Ciencias Biológicas, 2 (1): 38-42.

Botosaneanu, L. y J. H. Stock. 1982. Les Cyathura stygobies (Isopoda, Anthuridea) et sur tour delles des grandes et des pettits antilles. Bijdragen tot de Dierkunde, 15 (1):13-42.

Fernández, R. A. y M. Ortiz. 2004. Nota Científica. Nuevos registros de isópodos parásitos (Crustacea: Isopoda), de peces marinos (Actinopterygii) en aguas cubanas. Revista de Investigaciones Marinas, 25 (1): 73-74.

Fernández, R. A. y C. Varela. 2008. Registro nuevo de isópodo parásito (Crustacea, Isopoda) para Cuba. Cocuyo, 17: 11.

Fernández Osorio, R. A., R. I. Corrada Wong, P. P. Chevalier Monteagudo, H. Caballero Aragón y E. Cabrera Sansón. 2013. Primeros Registros Parasitológicos en Pez León, Pterois volitans (Linnaeus, 1758), para Aguas Cubanas. Proceedings of the 66th Gulf and Caribbean Fisheries Institute, November 4 - 8, Corpus Christi, Texas USA: 180-185.

Iliffe, T. A. y L. Botoşaneanu. 2006. The remarkable diversity of subterranean Cirolanidae (Crustacea, Isopoda) in the peri-Caribbean and Mexican Realm Bull. Institut Royal des Sciences Naturelles de Belgique. Biologie, 76: 5-26.

Lalana, R. y M. Ortiz. 1987. Redescripción y nueva localidad del isópodo Cleantis planicauda Benedict in Richardson, 1899 (Crustacea). Revista de Investigaciones Marinas, 8 (3): $23-28$.

Negoescu, I. 1968. Cyathura cubana n. sp. (Isopoda, Anthuridea) from the Caribbean Sea (Cuban waters). Extrait des Travaux du museum d'Histoire Naturelle G. Antipa, 10: $157-164$. 
Negoescu, I. 1982. A study of genus Cyathura from the Cuban freshwaters, with the description of a new cave species C. orghidani (Isopoda, Anthuridae). Resultats des Expeditions bioespeologiques Cubano-Roumanies a Cuba. Academia R. S. Romania, 4: 1-219.

Ocaña, F. 2009. Nuevos registros de crustáceos marinos (Amphipoda, Isopoda y Decapoda) de aguas cubanas. Revista de Investigaciones Marinas, 30 (3): 245-248.

Ortiz, M. y T. García. 1978. Isópodos parásitos de Micropogonia furnieri y Bairdiella ronchus (Pisces, Sciaenidae), en la Bahía de Cienfuegos. Ciencias, serie 8, Investigaciones Marinas, 38: 29-37.

Ortiz, M. y R. Lalana, 1980. Una nueva especie de isópodo (Crustacea, Isopoda), de los manglares de la costa sur de Cuba. Revista de Investigaciones Marinas, 1 (1): 160-174.

Ortiz, M. 1983. Guía para la identificación de los isópodos y tanaidáceos (Crustacea, Peracarida) asociados a los pilotes de las aguas cubanas. Revista de Investigaciones Marinas, $4(3): 3-24$.

Ortiz, M. y R. Lalana. 1990. Una nueva especie de isópodo (Crustacea, Isopoda), de los manglares de la costa Sur de Cuba. Revista de Investigaciones Marinas, 1 (2-3): 160-174.

Ortiz, M. y R. Lalana. 1993. Una nueva especie de isópodo (Isopoda, Asellota), asociado a Limnoria sp. (Isopoda, Flabellifera), de Cuba. Caribbean Journal of Science, 29 (1-2): 44-49.

Ortiz, M., A. García-Debrás y R. Lalana. 1998. Nueva localidad y descripción del macho de Cyathura orghidani Negoescu Vladescu. Revista de Investigaciones Marinas, (20) 2-3: 106-107.

Ortiz, M. 2001. Lista de invertebrados marinos, estuarinos y semiterrestres de la playa de Cojímar, en la costa norte de la provincia Ciudad de La Habana. Revista de Investigaciones Marinas, 22 (29): 93-102.

Ortiz, M., R. Lalana y C. Varela. 2002. Especie nueva del género Sphaeromopsis (Crustacea, Isopoda, Sphaeromatidae) de Cuba. Solenodon, 4: 1-5.

Ortiz, M., R. Lalana y C. Varela. 2002. Nuevos registros de anfípodos, isópodos y misidáceos (Crustacea: Peracarida), marinos cubanos. Revista de Investigaciones Marinas, 23 (2): 155-156.

Ortiz, M., R. Lalana, C. Varela y A. Ribot. 2002. Registros nuevos de crustáceos marinos bentónicos (Crustacea: Peracarida) para Cuba. Cocuyo, 12: 16.

Ortiz, M., R. Lalana y E. Suárez. 2003. Nuevos copépodos e isópodos (Crustacea) parásitos de peces del Archipiélago cubano, con la descripción de una nueva especie de copépodo. Avicennia, 16: 78-82.

Ortiz, M., R. Lalana y C. Varela. 2004. Una nueva especie del género Sphaeromopsis (Crustacea, Isopoda, Sphaeromatidae) de la plataforma sur oriental de Cuba. Solenodon, 4: 1-5. 
Ortiz, M., R. Lalana y C. Varela. 2006. Crustáceos no decápodos - Filo Arthropoda, Sub-filo Crustacea. Lista de especies registradas para Cuba. En Claro, R. (editor). Biodiversidad Marina de Cuba. CDR. Instituto de Oceanología, Ministerio de Ciencias, Tecnología y Medio Ambiente. La Habana, Cuba, ISBN: 978-959298-001-3.

Ortiz, M., R. Lalana, C. Varela, R. Arias y R. Cabrera. 2006. Registros nuevos de anfípodos e isópodos marinos cubanos (Crustacea, Peracarida). Cocuyo, 16: 19-20.

Ortiz, M., R. Lalana y C. Varela. 2007. Una nueva especie de isópodo espongícola (Isopoda, Flabellifera) de las aguas profundas del sur de Cuba. Avicennia, 19: 37-44.

Ortiz, M., R. Lalana y C. Varela. 2008. Registro nuevo del isópodo Synsynella choprae (Epicaridea, Bopyridae) para Cuba y primera consignación como parásito de la cámara branquial de Latreutes fucorum (Caridea, Hippolytidae). Cocuyo, 17: 12.

Ortiz, M. y R. Lalana. 2010. Claves para identificar a crustáceos cubanos (Arthropoda). Cocuyo, 18: 5-28.

[Recibido: 24 de mayo, 2018. Aceptado para publicación: 28 de junio, 2018] 